\title{
Berninis Löwengrube. Eine neue Deutung der Cappella Chigi in Santa Maria del Popolo
}

Als Gianlorenzo Bernini 1655 kurz nach dem Amtsantritt Alexanders VII. Chigi von dem neuen Papst den Auftrag bekam, zwei Skulpturen für die Chigi-Familienkapelle in Santa Maria del Popolo zu schaffen, trat er in einen doppelten Paragone ein: Zum einen mußte er sich als Bildhauer neben den ,Skulpturen eines Malers beweisen (hatte doch kein Geringerer als Raffael die Entwürfe für zwei bereits in der Kapelle befindliche Statuen Lorenzettos geliefert), zum anderen zwang ihn sein bislang ausschließlich in Malerei und Graphik dargestelltes Thema, Daniels Speisung durch Habakuk, zur Auseinandersetzung und Konkurrenz mit zweidimensionalen Vorbildern. Wie Bernini diese anspruchsvollen Aufgaben bewältigte ist Thema des vorliegenden Beitrags.

Zum Zeitpunkt der Auftragsvergabe kannte Bernini die Chigi-Kapelle schon genau, da er sie 1652-1654 für Kardinal Fabio Chigi (den späteren Papst Alexander VII.) restauriert hatte. In den ersten beiden Abschnitten des Textes möchte ich zeigen, daß die während des Pontifikats realisierten Arbeiten allerdings keineswegs das zuvor Begonnene bruchlos weiterführten: Im Gegenteil, Bernini dachte sich ein neues Konzept aus, das das bisher Geschaffene grundlegend uminterpretierte. Inwiefern er damit kreativ auf die Paragone-Situation reagierte, soll in Abschnitt 3 näher erläutert werden. Im vierten Abschnitt geht es dann um den sautoreflexiven Paragone bzw. um die Impulse, die von der Chigi-Kapelle auf Berninis Gestaltung des Alexandergrabes in St. Peter ausgingen.

\section{Das Kapellenprogramm vor 1655}

Die wechselvolle Geschichte der Kapelle, die der legendäre Bankier Agostino Chigi unter Leitung Raffaels in Santa Maria del Popolo errichten ließ, ist bereits oft Ge-

Dieser Text basiert auf dem Vortrag, den ich im Februar 2001 auf der Tagung Im Agon der Künste gehalten habe. Die zeitgenössischen Kapellenbeschreibungen, die ich damals nur kurz ansprechen konnte, wurden erstmals publiziert und ausführlich kommentiert in Bellori und Bernini rezipieren Raffael. Unbekannte Dokumente zur Cappella Chigi in Santa Maria del Popolo, wobei auch einige Gedanken des vorliegenden Aufsatzes aufgegriffen und weitergeführt wurden (Marburger Jahrbuch für Kunstwissenschaft, 30/ 2003). Für kritische Anregungen danke ich Ingo Herklotz und Tomaso Montanari.

1 Ich verweise insbesondere auf Cugnoni (1879-1881, 1883); Gnoli (1889); Shearman (1961); Bentivoglio (1984); Filippi (1984); Pinelli (2001); Riegel (2003). 


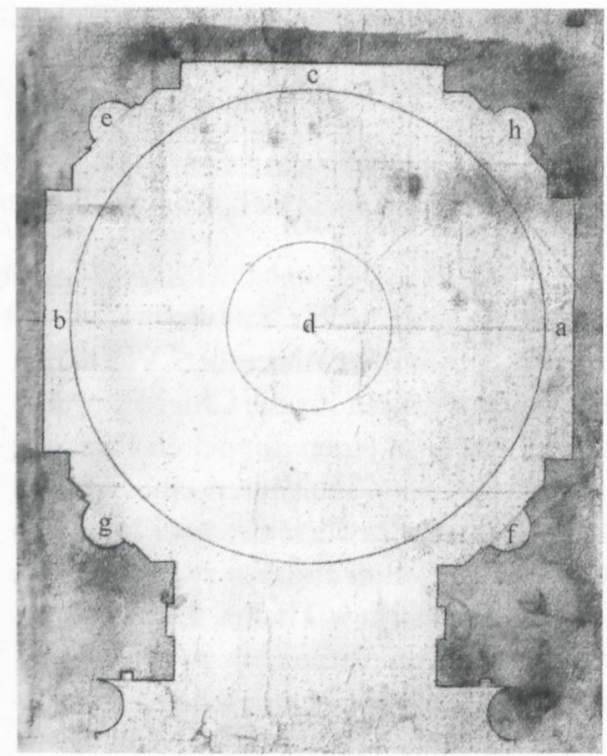

1. Das 1661 vollendete ikonographische Schema der Cappella Chigi in Santa Maria del Popolo, Rom: a = Grabpyramide des Agostino Chigi, darüber Lünettenbild von Raffaello Vanni, Die priesterlichen Vorfahren Mariens; $b$ = Grabpyramide des Sigismondo Chigi, darüber Lünettenbild von Raffaello Vanni, Die königlichen Vorfahren Mariens; $\mathrm{c}=$ Altar mit Antependium von Lorenzetto, Christus am Brunnen, darüber Altarbild von Sebastiano del Piombo und Francesco Salviati, Mariengeburt; $\mathrm{d}=$ Zugang zur Gruft mit opus-sectile-Darstellung eines wappentragenden Skeletts nach Entwurf von Gianlorenzo Bernini, darüber Kuppelmosaik von Aloisio De Pace nach Entwurf Raffaels, Gottvater und die acht himmlischen Sphären, sowie Gemälde im Kuppeltambour von Francesco Salviati, Die Schöpfungsgeschichte; e = Skulptur von Lorenzetto, Jonas, darüber Tondo von Francesco Salviati, Der Winter; $\mathrm{f}=$ Skulptur von Lorenzetto, Elias, darüber Tondo von Francesco Salviati, Der Sommer; $g$ = Skulptur von Gianlorenzo Bernini, Daniel, darüber Tondo von Francesco Salviati, Der Herbst; $\mathrm{h}=$ Skulptur von Gianlorenzo Bernini, Habakuk und der Engel, darüber Tondo von Francesco Salviati, Der Frühling

genstand kunsthistorischer Untersuchungen gewesen. ${ }^{1}$ Auch die von Fabio Chigi finanzierten Restaurierungsarbeiten in den Jahren 1652-1654 sind aufgrund von Briefen und Tagebuchnotizen des Auftraggebers eingehend geschildert worden. ${ }^{2}$ Ich werde daher im folgenden nur knapp darlegen, welche Ausgangssituation Fabio Chigi vorfand, welche Arbeiten er während seines Kardinalats unter Leitung Berninis durchführen ließ und wie das dadurch realisierte theologische Programm aussah.

Die von Raffael entworfene Chigi-Kapelle ist ein kleiner überkuppelter Zentralraum, in dem sich ursprünglich zwei pyramidenförmige Grabmäler gegenüberstehen sollten. Als Fabio Chigi die Kapelle 1626 bei seinem ersten Romaufenthalt besuchte und beschrieb, ${ }^{3}$ stand jedoch nur eine der Pyramiden (die rechte) an ihrem Ort (Abb. 1a). Sie war mit zwei Sockelreliefs dekoriert, besaß aber weder eine Inschrift noch ein Wappen und trug auch kein Porträt. Dennoch ging Fabio davon aus, es müsse sich um die Grablege des Kapellengründers Agostino Chigi handeln. ${ }^{4}$ Gegen-

2 Vor allem durch Hesse (1983); Petrucci (1998); Fabjan (1999).

3 Brief vom 26. Dezember 1626, abgedruckt bei Cugnoni (1881), S. $58 \mathrm{f}$.

4 Gnoli (1889), S. 320-322. Daß Fabio mit dieser Annahme irrte, hat Pinelli (2001) behauptet, worin ihm Riegel (2003) widersprach. Im folgenden wird mit Bezug auf die rechte Pyramide von "Agostinos" Grab die Rede sein, da es dies ja in den Augen des 17. Jahrhunderts zweifellos war. 
über befand sich das Grabmal des Kardinals Antoniotto Pallavicini, das anläßlich der Neugestaltung des Chores in die herrenlos scheinende Chigi-Kapelle versetzt worden war. ${ }^{5}$ In einem regen Briefwechsel diskutierte Fabio 1626-1629 mit seinem in Siena ansässigen Onkel Agostino, wie man dieses Grab entfernen und die Rechte der Familie auf die Kapelle wieder geltend machen könne. Da der junge Chigi 1629 als Vizelegat nach Ferrara entsendet wurde, geschah vorerst jedoch nichts. ${ }^{6}$

Nach einer steilen Diplomatenkarriere kehrte Fabio 1651 nach Rom zurück. Am 11. Dezember 1651 wurde er zum Segretario di Stato ernannt, am 19. Februar 1652 erhielt er die Kardinalswürde. Als Titelkirche erwählte er sich Santa Maria del Popolo und beauftragte wenig später Bernini mit der Vollendung der Familienkapelle. ${ }^{7}$ Die meisten der damals durchgeführten Arbeiten sind in einem viel zitierten anonymen Dokument aufgelistet. ${ }^{8}$ Die Kuppel wurde neu verbleit und durch größere Fenster besser beleuchtet. Raffaels Kuppelmosaiken wurden ebenso restauriert wie Salviatis Jahreszeiten-Tondi in den Zwickeln über den Statuennischen. ${ }^{9}$ Auf der linken Kapellenseite wurde das Pallavicino-Monument beseitigt und eine Grabpyramide für Sigismondo Chigi errichtet (Abb. 1b), während man das gegenüberliegende Grabmal restaurierte (Abb. 1a). ${ }^{10}$ Beide Pyramiden erhielten Bildnismedaillons der Verstorbenen sowie Inschriften, die vermutlich von dem päpstlichen Bibliothekar Lukas Holste entworfen worden waren. ${ }^{11}$ Über den Pyramiden gestaltete Raffaello Vanni zwei neue Lünettenbilder, ${ }^{12}$ deren Thema ein wohlinformierter Zeitgenosse, Benedetto Millino, als die königlichen und priesterlichen Vorfahren der Jungfrau Maria interpretierte. ${ }^{13}$ Ein großes, von Lorenzetto geschaffenes

5 Hesse (1983), S. 111; Fabjan (1999), S. 47.

6 Ebd., S. 47-49.

7 Petrucci (1998), S. 170, 182ff.; Fabjan (1999), S. 47, $49 \mathrm{ff}$.

8 Abgedruckt bei Cugnoni (1880), S. 439 f.

9 Ebd., S. 440; Petrucci (1998), S. 176.

10 Gnoli (1889), S. 321-323. Pallavicinos Grab gelangte in die angrenzende Kapelle: Millino, fol. 26v.

11 Petrucci (1998), S. 184-188; Angelini (1998), S. 138, 140; Fabjan (1999), S. 50; Petrucci (1999), S. 355. Alle Arbeiten an den Pyramiden waren 1653 abgeschlossen, wie aus dem Diario des Guiseppe Gualdi hervorgeht: „Il S.r Card.1 Ghisi perfettionò questo anno [1653] la sua capella al Popolo, fece un nuovo deposito in essa, e tanto in quello, quanto nel dicontro pose le statue, cioe teste, e busto di marmo bianco con inscrittioni di doi di sua casa." Herklotz (2003), S. 210.

12 Petrucci (1998), S. 176f. Laut Bentivoglio entstanden die Lünettenfelder überhaupt erst dadurch, daß Berrnini das hinter den Pyramiden entlanglaufende Gebälk einfügte: Bentivoglio (1986), S. 311f. Shearman (1981) sieht hingegen das offenbar nachträglich angelegte Gebälk noch als ein pentimento Raffaels.

13 Millino, fol. 30v. Diese Interpretation wurde von Weil-Garris Brandt unter Berufung auf Worsdale referiert, der Millinos Text offenbar kannte, ihn jedoch nicht weiter auswertete und seine Signatur nicht angab. Weil-Garris Brandt (1986), S. 152; Worsdale (1986), S. 92. In anderem Kontext verwies Ackermann (1996), S. 371, Anm. 3, auf Millinos Manuskript. Der Abschnitt über die Chigi-Kapelle liegt mittlerweile ediert vor: Strunck (2003), S. 178-180. 
Bronzerelief wurde von Agostinos Grab an den Altar versetzt und zum Antependium umfunktioniert (Abb. 1c). ${ }^{14}$ Den Altar erhöhte man um einige Stufen, die weiter als bisher in den Raum ragten. ${ }^{15}$ Die Öffnung im Boden, durch die man eine unter dem Altar befindliche Pyramide sehen konnte, wurde dabei geschlossen; statt dessen legte man im Zentrum der Kapelle einen kreisrunden Zugang zur Gruft an, den Bernini mit der opus-sectile-Darstellung eines wappentragenden Skeletts verschloß (Abb. 1d). ${ }^{16}$ Die hölzerne Kapellenschranke wurde durch eine Marmorbalustrade ersetzt; darüber verkündete am Kapellenbogen ein neues Wappen die Eigentümerrechte der Chigi. ${ }^{17} \mathrm{Zu}$ guter letzt wertete Fabio den Altar durch zusätzliche Reliquien auf und schmückte ihn mit einem Kruzifix, das gleichzeitig als Hostienbehälter diente, ${ }^{18}$ sowie mit sechs Bronzeleuchtern, die sich spätestens Ende 1654 in situ befanden. ${ }^{19}$ Die »Einweihung " der restaurierten Kapelle war bereits im Vorjahr zum Fest der Mariengeburt erfolgt (8.9.1653). ${ }^{20}$

Aufgabe einer Familienkapelle ist es, das Andenken der Verstorbenen zu wahren und ihr Seelenheil durch Messen zu sichern. Ewiger Ruhm der Familie und Auferstehung der Toten bilden das doppelte Ziel. Alle von Fabio veranlaßten Arbeiten in

14 Nobis (1979), S. 90-92; Fabjan (1999), S. 51. Das zweite, damals wohl eliminierte Bronzerelief des Pyramidensockels wurde publiziert von Bentivoglio (1986), S. 309, Tav. CXIX. Zur Ikonographie vgl. Anm. 28

15 Bentivoglio (1984), S. 128; Morello (1992), S. 193 und Abb. 3. Fabjan (1999), S. 51, 55, Anm. 45.

16 Frommel (1974), S. 344-348; Weil-Garris Brandt (1986), S. 152.

17 Fabjan (1999), S. 47, 49f. Das Wappen wurde 1652 angebracht, wie Giuseppe Gualdi in jenem Jahr in seinem Diario vermerkte: "Il Sig.r Card.I Ghisi Senese fece questo anno risarcire la capella de suoi antenati nella chiesa della Madonna del Popolo fatta con dissegno, e pitture di Raffael da Urbino, mà per il tempo era andato poco men, che affatto a male le pitture, e scoloriti li marmi, e posta sopra l'arco per di fuori l'arme della casa, la ridusse all'antica perfettione. "Herklotz (2003), S. 206-207.

18 Petrucci (1997), S. 188-190; Petrucci (1998), S. 189; Fabjan (1999), S. 51f., 55, Anm. 41.

19 Petrucci (1998), S. 196 (Tagebucheintrag Fabio Chigis vom 23. Dezember 1654).

20 Guiseppe Gualdi vermerkte unter dem 8.9.1653 in seinem Diario: „Natività della Beata Vergine Maria, festa solenne in molte chiese, et in particolare a S. Maria del Popolo, havendo il S.r Card.l Chigi titolare accomodata e perfettionata la capella della Natività della Madonna fatta da suoi antenati, dipinta di mano di Raffaele, et essendo ogni cosa rovinata fù fatta ripulire, e colorire, stuccare, e perfettamente raccomodare, havendo in oltre fatto far l'altra piramide sepulcrale, et in ambedue i ritratti di marmo di doi della casa. "Herklotz (2003), S. 228, Anm. 166. Über dasselbe Fest am 8.9.1653 schieb Giovan Battista Cancellotti 1661 in seinen Annales Mariani, die Kapelle sei damals "quasi erneut geweiht" worden: zitiert bei Delbeke (2001), S. 182, Anm. 49. Ich danke Maarten Delbeke für diesen freundlichen Hinweis. Kurz vor dem Fest, am 6.9.1653, berichtete Fabio Chigi seinem Neffen Agostino enthusiastisch: "So' al fine della Capp.a del Popolo riesce eccellente e pare un'altra mi costarà presso a 3000 di., ma gli meritava che val più di 30. Fabjan (1999), S. 50, 55, Anm. 40. Daß die Restaurierung der Kapelle 1654 bereits abgeschlossen war, geht auch aus einer Beschreibung vom 27.4.1654 hervor: abgedruckt bei Filippi (1984), S. 135. 


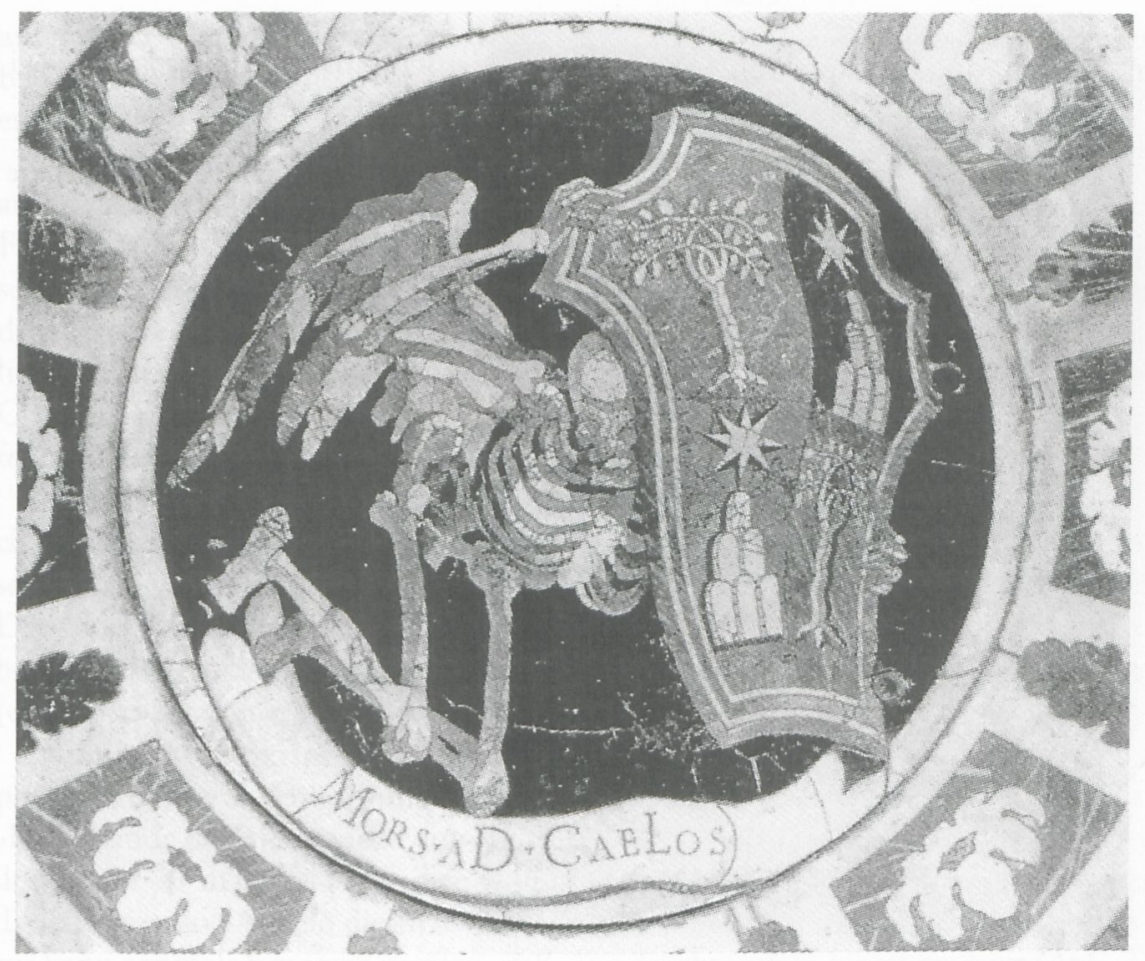

2. Gabriele Renzi (nach Entwurf von Gianlorenzo Bernini), Wappentragendes Skelett im Fußboden der Chigi-Kapelle, 1653/54. Opus-sectile-Arbeit aus Buntmarmor. Cappella Chigi, Santa Maria del Popolo, Rom

der Chigi-Kapelle ordnen sich in diesen Gedankenhorizont ein. Die Pyramiden, einer anonymen Kapellenbeschreibung zufolge Symbol für Unsterblichkeit, ${ }^{21}$ dienen mitsamt ihren Porträtmedaillons und Inschriften der weltlichen Memoria. Die Pyramidenspitzen ragen über das Gebälk hinaus und damit schon in die Zone der Heilsgeschichte hinein, die sich im Deckenbereich entfaltet. ${ }^{22}$ Raffaels Kuppelmosaiken zeigen laut Millino "i Cieli mossi dalle proprie Intelligenze, co' sette Pianeti, e col Firmamento. Nella Lanterna, Dio Padre in una Gloria d'Angeli, in atto di crear' il Mondo«. ${ }^{23}$ Die Schöpfung vollzieht sich dann in den kleinen Historienbil-

21 Chisiani Sacelli Descriptio, fol. 429v. Auf dieses Manuskript verwies Bentivoglio (1984), S. 129. Es liegt mittlerweile ediert vor: Strunck (2003), S. 173-178. Zahlreiche Indizien deuten darauf hin, daß es von Giovan Pietro Bellori ca. 1655 verfaßt wurde: ibid., S. 135-146.

22 Vgl. Melczer (1990), S. $165 f$.

23 Millino, fol. 29r. Moderne Deutungen dieser Mosaiken bei Shearman (1961), S. 140142 und Weil-Garris Brandt (1986), S. 128-145. 


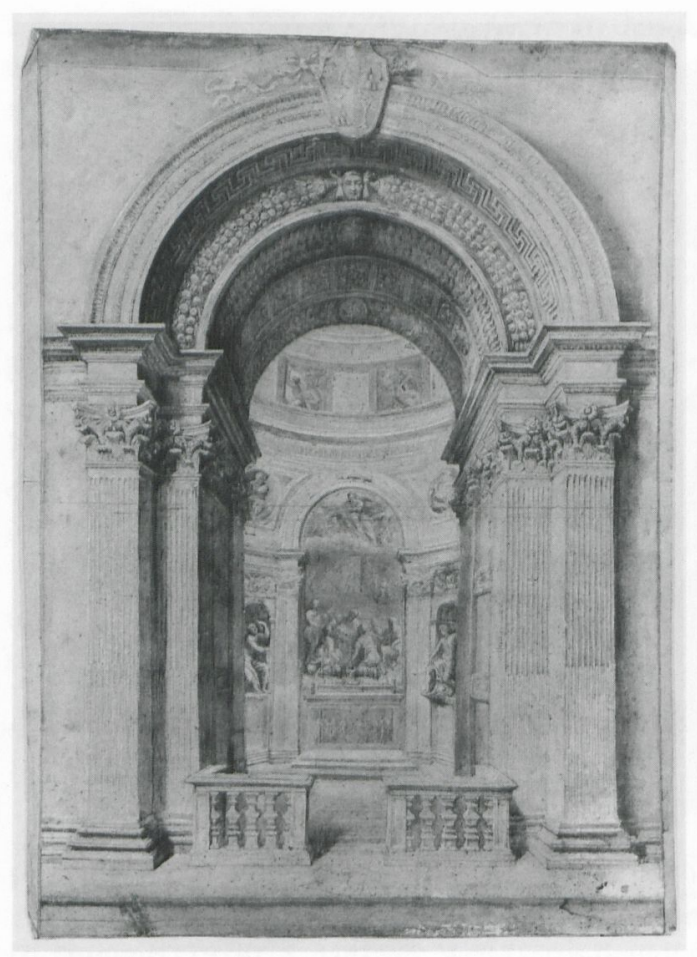

3. Gianlorenzo Bernini (Werkstatt), Blick in die Cappella Chigi in Santa Maria del Popolo, ca. 1654. Tinte und Aquarell auf Papier, $53,3 \times 37 \mathrm{~cm}$. Smith College Museum of Art, Northampton (Massachusetts)

dern der Tambourzone. Die königlichen und priesterlichen Vorfahren Mariens in den Lünetten fungieren als alttestamentarisches Bindeglied zwischen der Frühgeschichte der Welt in der Kuppel und dem christlichen Heilsgeschehen, das im Altarbild mit der Mariengeburt beginnt. Nach Ansicht eines anonymen zeitgenössischen Kommentators führen die Gestalten in den Lünetten vor, daß sowohl der geistliche als auch der weltliche Stand Gott anbeten solle. ${ }^{24} \mathrm{Da}$ die Figuren direkt über den Grabpyramiden angeordnet sind, scheinen sie für Agostino und Sigismondo Fürbitte zu halten. Was den Verstorbenen zu wünschen ist, manifestiert sich in dem opus-sectile-Bild des Fußbodens: Ein Skelett trägt das Chigi-Wappen aus der Gruft zum Himmel empor (Abb. 2). Stellvertretend für die Toten wird das Wappen dem Grab bzw. dem Vergessen entrissen. Diese Allegorie, die an eine antike Apotheose denken läßt, wird durch die Beischrift »Mors aD CaeLos [Iter] « zu einem Bild der Auferstehungshoffnung. ${ }^{25}$ So schließt Bernini den Kreis und macht

24 Chisiani Sacelli Descriptio, fol. 428v-429r. Abbildung der Lünetten bei Petrucci (1998), S. $176 f$.

25 Wie Millino, fol. 30v und die Chisiani Sacelli Descriptio, fol. 429r, überliefern, sollte die Inschrift ursprünglich "Mors aD CaeLos Iter« lauten, um durch die darin versteckten lateinischen Ziffern auf das Jahr MDCLI (1651) zu verweisen, in dem Fabio Chigi nach Rom zurückgekommen war. Die heutige Form der Inschrift (Abb. 2) geht aller- 
anschaulich, daß der segnende Gottvater im Zentrum der Kuppel nicht nur das Leben auf der Erde schuf, sondern nach dem Tod auch die Seelen wieder bei sich empfängt. ${ }^{26}$

Daß der Tod der Weg zum ewigen Leben ist (wie es ja die Fußbodeninschrift ausdrückt), wurde am Altar noch einmal besonders augenfällig. 1654 bemühte sich Fabio, die Kapelle zur Sakramentskapelle aufwerten zu lassen, und stiftete in diesem Zusammenhang ein Kruzifix, dessen Fuß als Hostienbehälter diente: ${ }^{27}$ Aus dem Kreuzeshügel, der 'Schädelstätter Golgatha, gewissermaßen aus der Grabeshöhle konnte man die Hostie herausnehmen, Sinnbild des auferstandenen Leibes Christi, Verheißung des ewigen Lebens, dessen der Gläubige durch die Meßfeier teilhaftig werden kann.

Auch die von Lorenzetto geschaffenen Skulpturen wurden auf die Leitthemen Eucharistie und Auferstehung bezogen. Das an den Altar versetzte Relief, das zwei Episoden aus dem Johannes-Evangelium vereint, "Eucharestiae pertinet mysterium «. ${ }^{28}$ Der vom Wal ausgespieene Jonas war als »Resurrectionis, atque immortalitatis symbolum« zu verstehen, während der von einem Engel gespeiste Elias als »Eucharestiae [quae, perpetua veneratione, in medio Altari colitur] argumentum « galt. $^{29}$

Millinos Manuskript bietet den wichtigen Hinweis, daß Lorenzettos zwei Prophetenfiguren zur Entstehungszeit des Textes (ca. 1656) den Altar flankierten: "Dalle bande dell'altare, nelle due nicchie delle quattro dette di sopra, son collocate due statue di marmo: l'una dice il Vasari essere d'Abacucco; ma io credo esser d'Elia, havendo l'Angelo dietro le spalle, e co' un vase d'acqua. ${ }^{30}$ Durch diesen Passus kann erstmals bewiesen werden, daß eine Zeichnung in Northampton (Abb. 3)

nach Rom zurückgekommen war. Die heutige Form der Inschrift (Abb. 2) geht allerdings nicht auf eine spätere Restaurierung zurück: vgl. Medici (1990). Das Wort "Iter" wurde bereits vor Realisierung des Schriftbandes gestrichen, aber bildlich durch den Flug des Skeletts ausgedrückt. Vielleicht wollte Fabio Chigi durch den verkürzten Text einen fiktiven Bezug zwischen der Kapellenrestaurierung und dem Heiligen Jahr MDCL (1650) herstellen.

26 Vgl. Hesse (1983), S. $114 \mathrm{f}$.

27 Bentivoglio, Valtieri (1976), S. 218f.; vgl. Petrucci (1997), S. 188-190; Petrucci (1998), S. 189; Fabjan (1999), S. 51 f.

28 Chisiani Sacelli Descriptio, fol. 426v. Photo des Reliefs bei Nobis (1979), Abb. 10. Während der linke Teil des Reliefs durch Christi Begegnung mit der Samariterin am Brunnen auf die Taufe verweist (Joh. 4, 14), besitzt der rechte Teil eucharistische Bezüge, da Christus die von seinen Jüngern angebotene Speise mit den Worten ablehnt: „Ich lebe von einer Speise, die ihr nicht kennt" (Joh. 4, 32). Vgl. Shearman (1961), S. 135 und Nobis (1979), S. 99.

29 Chisiani Sacelli Descriptio, fol. 426r.

30 Millino, fol. 29v. Zur Datierung von Millinos Text: Strunck (2003), S. 143-144. Vasari identifiziert den Elias übrigens korrekt: Vasari, hg. v. Milanesi (1906), Bd. 4, S. 558, 578. Die falsche Benennung kommt wohl von Alberici (1600), S. 16 oder von Landucci (1646), S. 29. Sie findet sich ferner in zwei Kapellenbeschreibungen aus den 1650er Jahren: abgedruckt bei Filippi (1984), S. 135. Daß die Statue als Elias gedacht war, geht aus einem Dokument von 1552 zweifelsfrei hervor: Nobis (1979), S. $246 f$. 


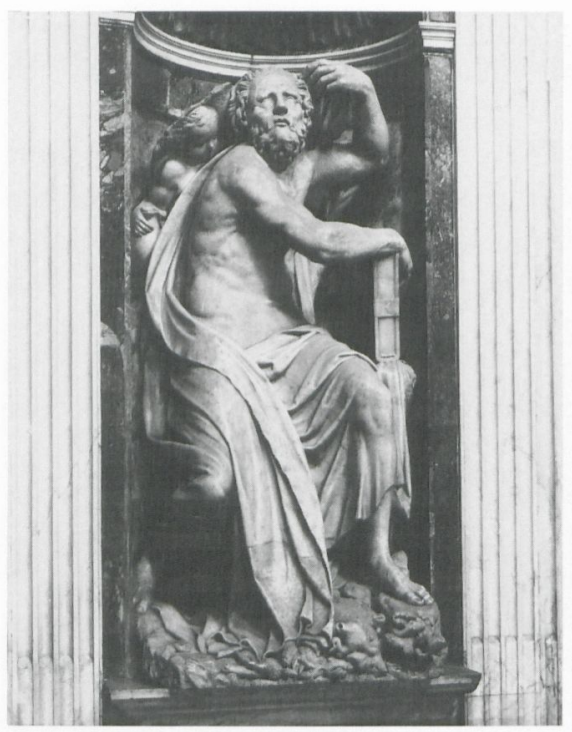

4. Lorenzetto (nach Entwurf von Raffael?), Elias, ca. 1518/20-1524. Skulptur aus Marmor. Cappella Chigi, Santa Maria del Popolo, Rom

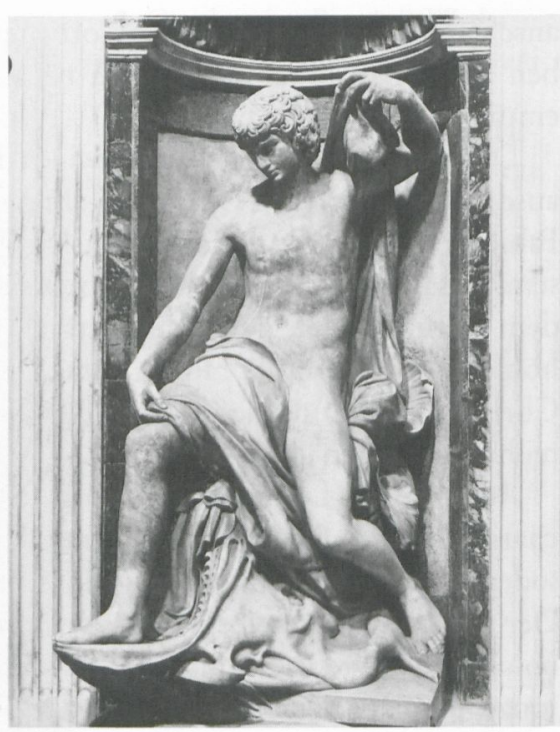

5. Lorenzetto (nach Entwurf von Raffael), Jonas, ca. 1516-1520. Skulptur aus Marmor. Cappella Chigi, Santa Maria del Popolo, Rom

den einst tatsächlich existierenden Zustand der Kapelle festhält und nicht bloß einen unausgeführten Entwurf Berninis dokumentiert. ${ }^{31}$ Die beiden Propheten, die Fabio 1626 in den Nischen zu Seiten des Eingangs gesehen hatte, ${ }^{32}$ müssen also im Laufe der Restaurierungsarbeiten an die Altarwand versetzt worden sein: Elias (Abb. 4) und Jonas (Abb. 5) wurden dort zu einem symmetrisch zum Altar gewandten Paar vereint.

Bei der bereits erwähnten »Einweihung« der Kapelle am 8.9.1653 empfand Fabio Chigi das Fehlen von zwei Skulpturen noch als Makel - wenig später spielte er mit dem Gedanken, je ein Werk bei Bernini und Algardi zu bestellen, doch scheinen ihn die zu erwartenden hohen Kosten abgeschreckt zu haben. ${ }^{33}$ Danach erst fiel möglicherweise die Entscheidung, die beiden existierenden Propheten zu ver-

$31 \mathrm{Zu}$ dieser Zeichnung vgl. Hesse (1983), S. 116; Wittkower (1997), S. 276; Fabjan (1999), S. 51f.; Petrucci (1999), S. 355; Ferrari, Papaldo (1999), S. 317.

32 Cugnoni (1879), S. 75. Bei diesem Arrangement handelte es sich wohl nicht um die ursprünglich intendierte Aufstellung. Eine Zeichnung des 16. Jahrhunderts zeigt den Jonas rechts des Altares: Shearman (1961), S. 131, Anm. 12. Erst ca. 1552 wurden Lorenzettos Skulpturen neben dem Kapelleneingang plaziert: Nobis (1979), S. 77. In dieser Position beschrieb sie Fabio Chigi 1626 und betonte dabei explizit, daß die beiden den Altar flankierenden Nischen leer seien: Cugnoni (1881), S. 58.

33 Fabjan (1999), S. 51 (speziell die Dokumente vom 13.9.1653). 
setzen. Wenn der Kardinal noch mit kunstvollen neuen Skulpturen gerechnet hätte, hätte er die prominenten Nischen neben dem Altar wohl nicht mit Lorenzettos Propheten besetzt. Bezeichnenderweise erachtete Millino die Kapelle als fertig ${ }^{34}$ auch ohne die beiden Skulpturen Berninis, die erst während Chigis Pontifikat in Auftrag gegeben wurden. Die heute in Northampton befindliche Zeichnung (Abb. 3) dürfte 1654 oder 1655 kurz nach Abschluß der Restaurierungsarbeiten entstanden sein, um das Werk des Kardinals zu verewigen. ${ }^{35}$

\section{Eine neue Deutung von Berninis Daniel und Habakuk}

Nach einer - wie er im Tagebuch vermerkte - schlaflosen Nacht wurde Fabio Chigi am 7. April 1655 zum Papst gewählt. ${ }^{36}$ Wenig später wandte er sich Santa Maria del Popolo mit neuer Energie und vor allem mit neuen Ressourcen zu. Er nahm die Restaurierung und Verschönerung der gesamten Kirche in Angriff, schmückte seine Familienkapelle weiter aus und bestellte für die beiden noch leeren Nischen zwei Skulpturen bei Bernini. ${ }^{37}$

Wann die Themen für die zwei fehlenden Werke festgelegt wurden, ist nicht bekannt ${ }^{38}$-auf jeden Fall aber vor dem 9. August 1656, denn an jenem Tag begegnen

34 Millino, fol. 7v: „Ultimamente Alessandro VII. Pontefice Massimo, doppo d'haver ridotta a perfezzione la cappella famosa della sua famiglia Chisia, fa abbellire ancor la Chiesa [...] «. In seiner Beschreibung der Kapelle erwähnt Millino dann nur die beiden Statuen Lorenzettos (fol. 29v).

35 Der von Wittkower und Hesse geäußerten Meinung, die Zeichnung müsse 1655 oder später datieren, da sie das Papstwappen zeige, ist nicht zuzustimmen, denn das Wappen wurde bereits 1652 am Kapellenbogen angebracht und ist nicht mit päpstlichen Insignien versehen. Siehe oben Anm. 17 sowie Hesse (1983), S. 116; Bentivoglio (1984), S. 137; Wittkower (1997), S. 276. Es wäre theoretisch möglich, daß das Blatt als Entwurf bzw. Präsentationszeichnung vor der Restaurierung der Kapelle angefertigt wurde; dies ist jedoch unwahrscheinlich, da die Darstellung eher einen Eindruck von der Kapelle insgesamt als von Berninis Umgestaltung gibt (die Gräber sind beispielsweise nicht zu sehen). Ich glaube daher, daß das Blatt 1654 nach Fertigstellung der Arbeiten entstand, um den damaligen (von Millino überlieferten) Zustand festzuhalten. Es wäre zu überlegen, ob die sehr detaillierte Zeichnung als Vorlage für einen Stich dienen sollte. Auf eine geplante Stichpublikation der gesamten Kapelle könnte auch der Umstand hindeuten, daß Carlo Cesi im Sommer 1653 Salviatis Tondi im Auftrag Fabios abzeichnete: vgl. Petrucci (1998), S. 176.

36 Petrucci (1998), S. 194.

37 Hesse (1983); Montagu (1989), S. 122-125, 134-140; Ackermann (1996).

38 Ostrow spekuliert unter Berufung auf Kauffmann, bereits Raffael könne Daniel und Habakuk für Santa Maria del Popolo vorgesehen haben, da er für die Chigi-Kapelle in Santa Maria della Pace ein fast identisches Prophetenprogramm realisiert habe (Daniel, Habakuk, Jonas und David): Kauffmann (1970), S. 222; Ostrow (1981), S. 169, Anm. 22. Fischel weist jedoch überzeugend nach, daß der angebliche Habakuk in Santa Maria della Pace ein Hosea ist, der bei einer späteren Restaurierung (durch Fabio Chigi?) um- 
"Daniel" und "Abacuc" erstmals in den Notizen des Papstes. ${ }^{39}$ Die beiden Propheten fügten sich optimal in das oben skizzierte theologische Programm ein: Die Geschichte von Habakuk, der dem in der Löwengrube schmachtenden Daniel Nahrung und Rettung bringt, konnte einerseits auf die Eucharistie, andererseits auf die Auferstehung bezogen werden. Habakuks Gabe wurde von Bosio als das Meßopfer gedeutet, das die Seelen im Fegefeuer stärke und dadurch ihr Leiden bzw. den Weg zur Auferstehung verkürze. ${ }^{40}$

Es ist dokumentarisch gesichert, daß der Daniel (Abb. 6) 1656 in Arbeit war und irgendwann zwischen dem 19. Mai und dem 26. Juni 1657 in seiner Nische links neben dem Kapelleneingang aufgestellt wurde (Abb. 1g). ${ }^{41}$ Da Jonas und Elias, wie oben dargelegt, damals zweifelsfrei zu Seiten des Altares standen, wäre für Habakuk die Nische rechts des Eingangs übriggeblieben. Ende 1661 wurde hingegen die heutige Anordnung realisiert, durch die Habakuk rechts neben den Altar gelangte und diagonal zu Daniel in Bezug gesetzt wurde (Abb. 1g und h). Das diesbezügliche Dokument spricht von Arbeiten "per porre in opera il gruppo della statua di Abacucch et altre due statue nella n.ra Cappella della Chiesa del Popolo, conf.e al conto agiustato dal Bernini«. Folglich fand damals nicht nur der Habakuk (Abb. 7) Aufstellung, sondern gleichzeitig wechselten auch Jonas und Elias ihre Position: Jonas, den die Zeichnung in Northampton (Abb. 3) rechts des Altares dokumentiert, erhielt seinen heutigen Platz auf der linken Seite der Altarwand, während Elias von dort in die Nische rechts des Eingangs wanderte ${ }^{42}$ - eine ziemlich um-

benannt wurde: Fischel (1948), Bd. 1, S. 181f. Weil-Garris Brandt (1986) meint, Daniel und Habakuk könnten bereits im 16. Jahrhundert vorgesehen gewesen sein, da sie zusammen mit Jonas und Elias auf die vier Elemente verweisen und sich so in das kosmologische Gesamtprogramm einordnen (S. 154f.). Ein solches, in der mündlichen Überlieferung noch präsentes Programm erklärt vielleicht, warum Lorenzettos Elias vor der Bestellung von Berninis Skulpturen wiederholt als Habakuk angesprochen wurde (siehe oben, Anm. 30). Fabio Chigi selbst scheint sich jedoch bezüglich der Komplettierung des Statuenzyklus nicht sicher gewesen zu sein, da er ungefähr 1653 den Rat von Lukas Holste anforderte: Fabjan (1999), S. 51.

39 Krautheimer, Jones (1975), S. 203, Nr. 30; vgl. Morello (1981), S. 322.

40 Bosio (1650), S. 600f.; Messerer (1962), S. 294; Hesse (1983), S. $122 f$.

41 Hesse (1983), S. 116.

42 Die Quelle von 1661 ist abgedruckt bei Golzio (1933/34), S. 139f. Vermutlich erfolgte der Planwechsel bereits im Jahr 1658, in dem der Papst notierte: "Cav. Bernino = le spese per la Chiesa del Pop(ol)o [...] e circa il mettere sù le due statue". (Tagebucheintrag vom 4.9.1658, abgedruckt bei Krautheimer, Jones (1975), S. 209, Nr. 236; vgl. Morello (1981), S. 325.) Dieser Passus wird gelegentlich als Beleg dafür gewertet, daß der $\mathrm{Ha}$ bakuk 1658 schon zur Aufstellung bereit war. Wieso hätte man mit seiner Plazierung dann aber bis Ende 1661 gewartet? Da der Daniel ja bereits 1657 in seiner Nische stand, hat die Formulierung "mettere sù le due statue« 1658 nur dann Sinn, wenn "mettere sù " nicht den simplen Aufstellungsprozeß, sondern ein Aufstellungskonzept meint. Meiner Meinung nach begann Bernini überhaupt erst mit dem Meißeln des Habakuk, nachdem 1658 die Entscheidung für eine diagonale Anordnung gefallen war. Die wenigen dokumentarischen Belege zu diesem Werk (aufgeführt bei Hesse (1983), S. 116) widerspre- 


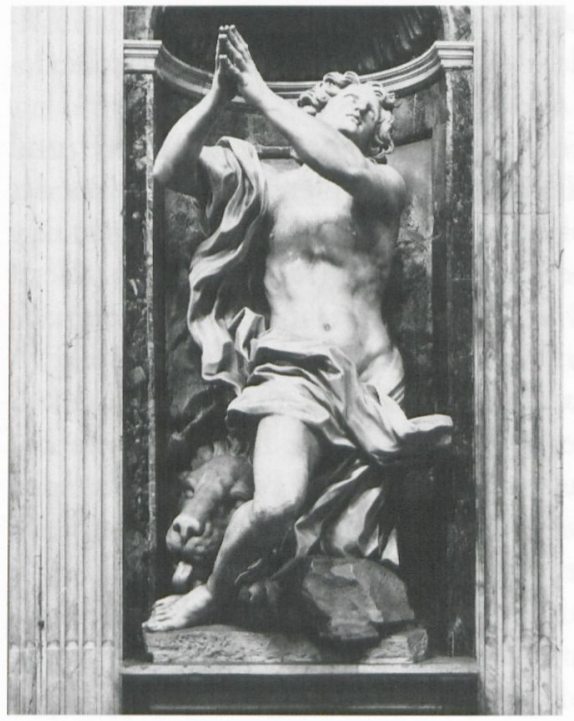

6. Gianlorenzo Bernini, Daniel, ca. 1656-1657. Skulptur aus Marmor. Cappella Chigi, Santa Maria del Popolo, Rom.

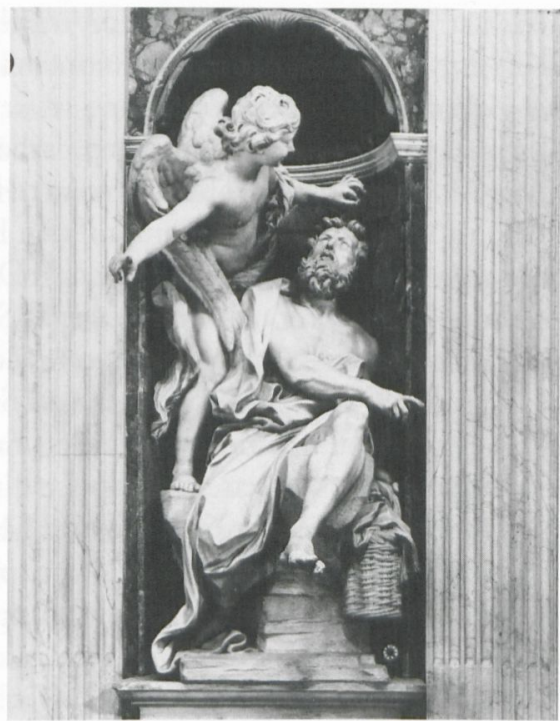

7. Gianlorenzo Bernini, Habakuk und der Engel, ca. 1658-1661. Skulptur aus Marmor. Cappella Chigi, Santa Maria del Popolo, Rom.

ständliche Aktion, die zeigt, daß der dadurch erzeugten neuen räumlichen Beziehung zwischen den Figuren große Bedeutung beigemessen wurde.

Die diagonale Gegenüberstellung von Daniel und Habakuk, die Bernini bereits in den Gestaltungsprozeß einkalkulierte (denn die weisende Geste des Engels ist auf einen Adressaten schräg gegenüber ausgerichtet), ermöglichte eine raumübergreifende erzählerische Beziehung zwischen Daniel und Habakuk. Immer wieder wird die Ansicht wiederholt, Bernini habe den Moment gestalten wollen, in dem der Engel in Juda an Habakuk herantrete und ihm bedeute, Daniel Hilfe nach Babel zu bringen; im nächsten Augenblick werde der Engel Habakuk an den Haaren zu Daniel hinübertragen, der in der Löwengrube inbrünstig um Rettung bete. ${ }^{43}$

chen einer solchen Interpretation nicht: Zwar wurde 1656 der Marmorblock angeliefert und der Papstneffe Flavio Chigi zu Bernini geschickt »a dimandar dell'Abacuc», doch läßt sich daraus nicht folgern, daß Bernini damals schon über bloße Vorstudien hinausgelangt war - zuerst stellte er ja den Daniel fertig, und außerdem war er mit zahlreichen anderen päpstlichen Aufträgen beschäftigt.

43 Soweit ich sehe, wurde diese Ansicht erstmals von Benkard (1926, S. 28f.) vertreten. Frühere Monographien kommentieren das Verhältnis der Skulpturen zueinander nicht: Reymond (o. J.), S. 123-126; Fraschetti (1900), S. 279-281; Pollak (1909), S. 98f.; Boehn (1912), S. 89; Munoz (1925), S. 23f. Benkards Meinung folgten: Wittkower (1955), S. 10; Shearman (1961), S. 152; Hibbard (1965), S. 187; Kauffmann (1970), 
Doch stimmt diese Interpretation überhaupt? Woran läßt sich erkennen, daß $D a$ niel und Habakuk sich an weit voneinander entfernten Orten in zwei verschiedenen Ländern befinden?

Betrachtet man die ikonographische Tradition des zuvor nur in Malerei und Graphik geschilderten Themas, so wird klar, daß sich die italienischen Künstler des 16. und 17. Jahrhunderts ganz auf den Höhepunkt der Geschichte, die Begegnung in der Löwengrube, konzentrieren. ${ }^{44}$ Dabei können verschiedene Akzente gesetzt werden. Paris Bordone und Giuseppe Salviati zeigen, wie Daniel die Hände nach der Nahrung ausstreckt, die der von einem Engel getragene Habakuk ihm bringt. ${ }^{45}$ Giovanni Bilivert, Salvator Rosa und Sebastiano Mazzoni thematisieren ebenfalls die Übergabe des Korbes, lassen den Engel aber gen Himmel deuten, um auf die göttliche Quelle der rettenden Speise hinzuweisen. ${ }^{46}$ Während Biliverts Daniel den Korb ergreift, erhebt er bei Rosa und Mazzoni die Arme in einem Gestus des überwältigten, dankbaren Glücks. Dieser spirituelle Aspekt findet sich auch bei Cigoli und Novelli: Statt sich auf die Nahrung zu stürzen, breitet Daniel die Arme in einem Inbrunstgestus erstaunt und dankbar aus. ${ }^{47}$ Pietro da Cortonas Version des Geschehens basiert klar auf Cigolis Vorbild: Daniel schaut nicht etwa auf die Speisen, sondern blickt ergriffen himmelwärts. ${ }^{48}$ Federico Zuccaris Fresko in Il Gesù (Abb. 8) pointiert schließlich Daniels Dankbarkeit noch mehr. Habakuk ist hier bereits in der Grube gelandet; doch würdigt Daniel ihn keines Blickes, sondern wendet sich betend zum Himmel. ${ }^{49}$

In vollplastischer Skulptur war die Geschichte von Daniels Rettung noch nicht verbildlicht worden - Daniel und Habakuk begegnen vor Bernini allenfalls als isolierte Prophetenstandbilder. ${ }^{50}$ Es gab jedoch ein wichtiges Vorbild für über den

S. 229f.; Hesse (1983), S. 118f.; Schulze (1989), S. 184; Schmitt (1997), S. 233; Avery (1998), S. 153f.; Angelini (1998), S. 141; Angelini (1999), S. 52; Fabjan (1999), S. 52ff. 44 Andere Auffassungen des Themas finden sich meines Wissens nur außerhalb Italiens: Judson (1970), S. 121f., Abb. 30. Lanfrancos für San Paolo fuori le mura bestimmtes Gemälde, das laut Bellori Habakuk und den Engel vor der Abreise zeigt, ist von Schleier überzeugend als Darstellung des Elias identifiziert worden: Schleier (1965), S. 68, 358f., 363; Schleier (1970), S. 4, Abb. 1. Etwas enigmatisch bleibt das Fresko in Caprarola, auf dem Daniel und Habakuk sich zwar beide in der Löwengrube befinden, einander aber den Rücken zuwenden: Portoghesi (1996), Tav. LXXXVI. Darstellungen, die Daniel allein in der Löwengrube abbilden, beziehen sich auf ein früheres Ereignis seines Lebens (Daniel 6, 16-24), das nicht mit dem zweiten Aufenthalt in der Löwengrube verwechselt werden darf (Daniel 14, 30-42).

45 Martini (1968), S. 22, Abb. 1; McTavish (1981), S. 128ff., Abb. 150.

46 Ivanoff (1947), S. 42ff., Abb. 43; Peroni (1995), Bd. 2/2, S. 753, Abb. 1553; Volpi (1998), S. 358-360, Abb. 2.

47 Natoli (1963), S. 173, Tav. LXV, Abb. 9f.; Merz (1991), S. 20, Anm. 77, Abb. 2.

48 Merz (1991), S. 17-20, Farbtafel 1.

49 Kauffmann (1970), S. 223, Abb. 121; vgl. auch Stechow (1964), Plate 31.

50 Beispiele: Donatellos Habakuk (Florenz, Museo dell'Opera del Duomo) und Alessandro Vittorias Daniel (Venedig, San Giuliano). Vgl. Pope-Hennessy (1993), S. 60-62 und Kauffmann (1970), S. 223, Abb. 122. Auch die inschriftlich als Daniel und Habakuk 
Raum hinweg kommunizierende Figurenpaare: Verkündigungsgruppen. ${ }^{51}$ Sowohl in der Skulptur als auch in der Malerei Italiens kam es vor, daß Maria und der Engel an zwei räumlich getrennten Orten plaziert wurden, z. B. beidseitig neben einem Durchgang. ${ }^{52}$ Trotzdem war jedem Kirchgänger klar, daß die Jungfrau und der ihr gegenüberstehende himmlische Botschafter in einem räumlichen und zeitlichen Kontinuum agierten.

Vor diesem Hintergrund wird deutlich, mit welchen Sehgewohnheiten und Erwartungshaltungen das Publikum des 17. Jahrhunderts die Chigi-Kapelle betrat. Wenn zwei Skulpturen trotz räumlicher Distanz miteinander korrespondierten (wie das Daniel und Habakuk durch die zeigende Vermittlerfigur des Engels ja überdeutlich tun), dann ging man davon aus, daß sie zeitlich und räumlich in direktem Kontakt zueinander stünden (wie bei einer Verkündigungsgruppe). Und wenn man erkannte, daß es sich bei den Skulpturen um Daniel und Habakuk handelte, dann erwartete man aufgrund der ikonographischen Tradition, daß ihr Zusammentreffen in der Löwengrube bzw. Daniels Dankgebet dargestellt sei.

Natürlich ist es Gianlorenzo Bernini zuzutrauen, daß er solche traditionsgeprägten Wahrnehmungsmuster bewußt unterlief und gerade das zeigen wollte, womit der Betrachter nicht rechnete. Der etablierten Forschungsmeinung zufolge hätte er in der Chigi-Kapelle etwas höchst Ungewöhnliches getan, nämlich zwei Szenen zusammenmontiert, die zwar zur selben Zeit, aber an völlig verschiedenen Orten abliefen, bevor die Protagonisten einander begegneten. Das zentrale Ereignis der Geschichte, die gottgewollte heilsbringende Tat, läge demnach noch in der Zukunft. Aber hätte das in einer Kapelle Sinn? Und bestätigen die Skulpturen selbst diese Auslegung?

Die auf Selbstzeugnissen Gianlorenzos beruhenden Viten von Domenico Bernini und Filippo Baldinucci geben leider keine Hinweise zur Deutung der Gruppe. ${ }^{53}$ Eine Interpretation muß daher vom anschaulichen Bestand ausgehen. Die herr-

bezeichneten Propheten, die Raffael an der Fassade der Chigi-Kapelle in Santa Maria della Pace darstellte, sind statuenhaft isolierte Einzelfiguren, die in keinerlei narrativem Verhältnis zueinander stehen: Fischel (1948), Bd. 1, S. 181-183 und Bd. 2, Abb. 194, 196; vgl. oben Anm. 38.

51 Hesse (1983), S. 120, verweist dazu eigentümlicherweise nur auf den Regensburger Dom.

52 Einige willkürlich herausgegriffene Beispiele aus der italienischen Malerei und Skulptur: Arasse (1999), S. 23, 30, 80f., 85f., 165-167, 184f., 270f.; Choppy (1991), S. 24f; Kruft (1976), S. 30, Abb. 24; Kruft (1980), Tafel 456, 477; Kruft (1995), Tafel 30, 65; Poeschke (1990), Tafel 186, S. 195, Abb. 90; Radcliffe, Baker, Maek-Gérard (1992), S. 146-149; Bock (2001), S. 33, Abb. 7 und S. 38, Abb. 12.

53 Domenico Bernini (1999), S. 107, interpretiert die Beziehung der beiden Figuren nicht: "due bellissime Statue di mano del Cavaliere, una delle quali rappresenta Habacùc preso pe' capelli dall'Angelo, l'altra Daniele frà i Leoni«. Baldinuccis Lebensbeschreibung nennt nur die Titel der Skulpturen: Baldinucci, hg. v. Ludovici (1948), S. 108, 110. Zu diesen Viten, die noch zu Lebzeiten Berninis auf dessen Initiative hin begonnen wurden, vgl. Montanari (1998), S. 400-405. 
schende Forschungsmeinung stützt sich vor allem auf die Gesten Habakuks und des Engels (Abb. 7): Der Engel zeige Habakuk, wohin er ihn gleich davontragen werde, während Habakuk andeute, daß er eigentlich in die entgegengesetzte Richtung gehen wolle. ${ }^{54}$ Allerdings weist er dabei auch auf das Grab des Agostino Chigi, das sich direkt neben ihm befindet (Abb. 1a und h). Wie Messerer betonte, fand Habakuk deswegen neben dem Altar Aufstellung, weil die Speisen in seinem Korb das eucharistische Mahl symbolisieren konnten. So wie Habakuk Daniel speiste, so stärke die Eucharistie die Seelen der Toten und verkürze dadurch ihre Zeit im Fegefeuer, meinten die Theologen des 17. Jahrhunderts. ${ }^{55}$ Dementsprechend ist Habakuks Geste vielleicht gar nicht narrativ gemeint, sondern soll den theologisch bedeutsamen Bezug zwischen Habakuk, dem Korb, der Eucharistie und dem Verstorbenen veranschaulichen.

Habakuk und der Engel sind diejenigen Skulpturen, die dem Betrachter als erste auffallen, wenn er sich vom Kircheneingang aus (d. h. von schräg links) der Cappella Chigi nähert. Die weit ausgreifenden Gesten der Figuren dürften daher auch ästhetischem Kalkül entsprungen sein: Sie wecken Neugier, indem sie auf Ausstattungselemente verweisen, die auf den ersten Blick nicht sichtbar oder optisch weniger prominent sind. Speziell der Fingerzeig des Engels fordert dazu heraus, näher zu treten, um den Sinn der rätselhaften Gruppe zu entschlüsseln. Da faßt ein Engel einen Mann an den Haaren - ein höchst seltsamer, ja unerhörter Anblick in einer Kirche! Erst wenn man dem Fingerzeig des Engels mit den Augen folgt, entdeckt man den neben dem Eingang vversteckten Daniel und kann sich die Geschichte zusammenreimen. ${ }^{56}$

Die Geste des Engels dient dazu, einen narrativen Bezug zwischen den beiden Nischen herzustellen. Das bedeutet nicht zwangsläufig, daß sie Habakuk signalisiert >dorthin werde ich dich bringen . Wie ein 1629 entstandenes Gemälde von Pietro Novelli belegt, macht dieser Fingerzeig nämlich auch dann noch Sinn, wenn Habakuk schon an der Löwengrube eingetroffen ist: 'Schau, das ist Daniel, dem du helfen sollst ${ }^{57}{ }^{57}$

Die schriftliche Überlieferung berichtet, Habakuk sei von dem Engel überrascht worden, als er aufs Feld ging, um den Schnittern ein Mahl zu bringen. ${ }^{58}$ Berninis

54 Wittkower (1955), S. 10 und Nachfolge (s. Anm. 43).

55 Messerer (1962), S. 294 f.

56 Vgl. Schmitt (1997), S. 239.

57 In Novellis Gemälde schweben der Engel und Habakuk direkt über der Löwengrube, wobei der Engel auf Daniel weist: Natoli (1963), S. 173, Tav. LXV, Abb. 10. Novellis Komposition wurde von Guglielmo Cortese im Refektorium von San Carlo ai Catinari variiert - vielleicht hatte Bernini dieses römische Vorbild vor Augen. Die Datierung von Corteses Werk ist allerdings unklar: Graf, Schleier (1973), S. 798, Abb. 39.

58 Die Erzählung von Daniel und Habakuk findet sich in der Septuaginta, einer Handschrift, die einen der Schätze der Chigi-Bibliothek bildete. Der Text, dessen Authentizität von den Protestanten bestritten wurde, war 1548 auf dem Konzil von Trient für kanonisch erklärt worden und lag daher in der von Pius V. und Clemens VIII. besorgten Ausgabe der Vulgata zu Berninis Zeit schon lange gedruckt vor (Daniel 14, 30-42): Bi- 
Habakuk geht jedoch nicht, sondern sitzt auf einem Fels. Daniel kniet ebenfalls auf einem Stein. Schon oft wurde beobachtet, daß die felsige Auskleidung von Daniels Nische den Ort als Löwengrube kennzeichnen soll. ${ }^{59}$ Die Schlußfolgerung liegt nahe, Habakuk befinde sich an demselben felsigen Ort: Der Engel setzt ihn gerade in der Löwengrube ab. Verwirrt und benommen von seiner wundersamen Flugreise staunt Habakuk den Engel an.

Der biblische Text erwähnt nicht, daß Daniel um Rettung gefleht habe, paraphrasiert aber sein Dankgebet nach Habakuks Eintreffen. Insofern ist nicht recht nachvollziehbar, warum die gesamte Bernini-Literatur Daniels Geste als eine Bitte um Rettung interpretiert. Sein Gesichtsausdruck wirkt eher ruhig und beseligt, zumal ja der Löwe bereits besänftigt an Daniels Fuß leckt (Abb. 6).

Da Bernini vierzig Jahre lang jede Woche mindestens einmal in der Jesuitenkirche Il Gesù gebetet haben soll, ${ }^{60}$ muß ihm Federico Zuccaris dortiges Fresko der Begegnung von Daniel und Habakuk (Abb. 8) bestens vertraut gewesen sein. Ich vermute, daß es seine Konzeption der Skulpturen für die Chigi-Kapelle unmittelbar inspirierte: Ebenso wie im biblischen Text bzw. wie bei Zuccari wendet sich Berninis Daniel dankend an den (im Kuppelmosaik dargestellten) Schöpfer, der ihm Habakuk zu seiner Rettung in die Löwengrube geschickt hat.

Diese Interpretation der Figuren harmoniert auch mit der zeitgenössischen Bibelauslegung. Der Jesuitengeneral Gian Paolo Oliva, der sowohl zu Bernini als auch zu dessen Auftraggeber Alexander VII. enge Kontakte unterhielt, kommentierte die Geschichte von Daniel und Habakuk wiederholt. 1659 und 1664 veröffentlichte er in zwei von Alexander persönlich eingeleiteten Bänden die Predigten, die er im Palazzo Apostolico gehalten hatte. Bernini kannte die Texte offenbar, da er sie 1665 einem jungen Mann zur Lektüre empfahl. ${ }^{61}$ In einer 1664 erschienenen Predigt, die durchaus einige Jahre früher verfaßt worden sein könnte und also möglicherweise in direktem Zusammenhang mit der Fertigstellung der Chigi-Kapelle (1661) steht, erzählte Oliva die Geschichte von Daniel und Habakuk und hob dabei besonders Daniels Dankbarkeit hervor: »Indi rizzatosi, nel toccare il primo boccone del vaso rusticano, esclamò molle di lagrime e stupefatto delle divine misericordie: Recordatus es mei Deus! ${ }^{62}$ In einer späteren Predigt präzisierte Oliva, Daniel habe vor dem Essen gebetet: »Alzò le mani inverso al Cielo; e, prima di pascersi, disse all'eterno Padre: Voi così amorosamente riflettete à mè, che nulla merito? Et ait Daniel: Recordatus es mei Deus? « ${ }^{63}$ In der Vulgata heißt es: „Et ait Daniel: Recordatus es mei, Deus; et non de-

blia Sacra (1965). Vgl. Wittkower (1955), S. 10; Ostrow (1981), S. 168, Anm. 11 und S. 169, Anm. 20; Fabjan (1999), S. 52.

59 Z. B. Hesse (1983), S. 120.

60 Lavin (1998), S. 18, 21.

61 Chantelou (2001), S. 85 (23. Juli 1665). Zu den Kontakten zwischen Bernini und Oliva siehe Kuhn (1993) und Montanari (1997), $46 \mathrm{f}$.

62 Oliva (1664), S. 561.

63 Oliva (1674), Bd. 3, S. 663. 


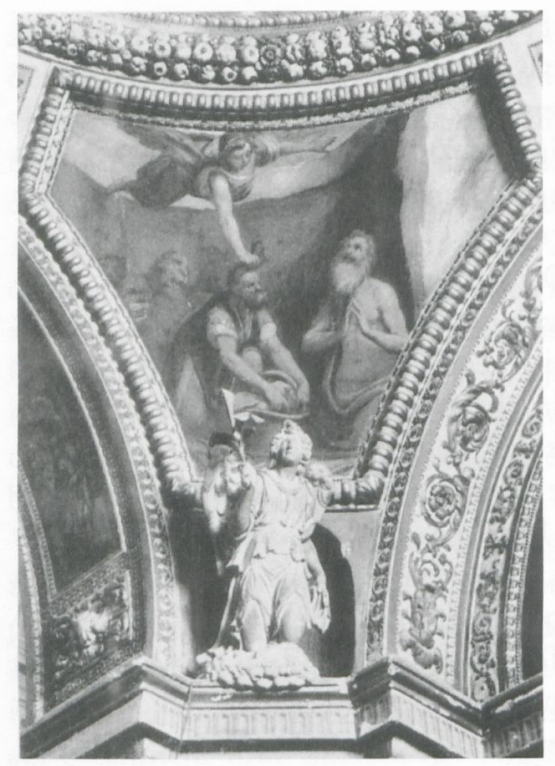

8. Federico Zuccari, Daniel

und Habakuk, ca. 1598-99. Fresko.

Cappella degli Angeli, Il Gesù, Rom.

reliquisti diligentes te. ${ }^{64}$ Diese Dankesworte Daniels sind wohl die Aussage, an die man sich in der Chigi-Kapelle erinnern sollte (und an die auch Oliva in seinen Predigten erinnerte): Der Herr hat an Daniel gedacht; er läßt die Gläubigen nicht im Stich, sondern schickt dem Rettung, der in Not ist. ${ }^{65}$

Alexander VII. dürfte als Berninis Auftraggeber darauf geachtet haben, daß eine theologische Botschaft vermittelt wurde, die sowohl in der Bibel als auch in der Bibelauslegung und in bildlichen Darstellungen der biblischen Geschichte fest verankert war. Wieso hätte Alexander eine Gruppe in Auftrag geben sollen, die vom biblischen Bericht abwich und nicht die Gewißheit der Rettung thematisierte, sondern das im Text gar nicht erwähnte Flehen um Rettung? Die Botschaft, daß Gott sich an Daniel erinnerte und ihm bereits geholfen hat, paßte zudem viel besser in das optimistische theologische Konzept der Chigi-Kapelle, die mit Jonas und Elias ja bereits zwei andere von Gott gerettete Propheten aufwies.

Das Neue und Besondere von Berninis Skulpturen besteht folglich nicht in der Wahl des dargestellten Moments; innovativ ist vielmehr, wie das Ensemble den Betrachter in das Geschehen einbezieht. Wenn Daniel und Habakuk sich nicht (wie gemeinhin angenommen) an zwei weit voneinander entfernten Orten in Juda bzw.

64 Daniel 14, 37.

65 Eine etliche Jahre nach der Chigi-Kapelle entstandene Mosaikdekoration in St. Peter reiht Daniel und Habakuk unter lauter lobpreisende Gestalten ein und bestätigt damit, daß der Dank an Gott als die theologische Essenz der Geschichte gesehen wurde: Pinelli (2000), Textband, S. 549-551. 
Babel befinden, sondern beide in der Löwengrube sind, dann ist der Kapellenbesucher mitten unter ihnen. Der ideale Betrachterstandpunkt liegt an der Diagonale, die zwischen den beiden Figuren verläuft. Die Geste des Engels greift in die Sphäre des Rezipienten hinein und bindet ihn gewissermaßen in die raumübergreifende Kommunikation der Propheten ein. So wird der Gläubige dazu angeregt, Daniels Erleben zu seiner eigenen Realität in Bezug zu setzen. Er selbst schmachtet in der Höhle des Todes, ebenso wie Daniel und wie jeder Sterbliche; doch kann er auch wie Daniel durch die Eucharistie Stärkung erfahren.

Der Planwechsel, durch den Daniel und Habakuk einander gegenübergestellt wurden, zerstörte nicht bloß die zuvor existierende Anordnung der Skulpturen, um eine narrative $>$ Brücke zwischen Berninis Werken zu schlagen, sondern veränderte das Raumkonzept in einem viel tiefergehenden Sinne. Die während Fabio Chigis Kardinalat ausgeführten Restaurierungsarbeiten hielten noch an der originalen Unterteilung der Kapelle in drei Zonen fest: oben in der Kuppel Gottvater im Himmel, im Mittelbereich der Mensch, in der Gruft der Tod. Das im Zentrum des Fußbodens eingelassene opus-sectile-Skelett (Abb. 2) scheint zwar aus der Gruft emporzufliegen, stellt aber keine Bedrohung dar, sondern 'rettet im Gegenteil das Chigi-Wappen. Das nach 1655 realisierte Raumkonzept durchbrach Raffaels DreiZonen-Schema, indem es die beruhigende Grenze zwischen Gruft und Lebenswelt aufhob: Auf einmal fand sich der Kapellenbesucher mitten in der Löwengrube wieder, die traditionell als Sinnbild für das Grab ausgedeutet wurde. ${ }^{66}$ Alexander VII. und Bernini, die beide ständig über ihren bevorstehenden Tod meditiert haben sollen, ${ }^{67}$ wollten dem Kirchgänger offenbar die Erkenntnis vermitteln, daß jeder Erdenmensch schon in der Gruft wandelt - Erlösung bzw. wahres Leben kann nur von Gott in Gestalt der Eucharistie kommen.

\section{Paragone in der Chigi-Kapelle}

Das Kräftemessen mit Michelangelo und mit der Antike sowie der Wettstreit mit der Malerei bilden Grundkonstanten in Berninis Euvre, ${ }^{68}$ die auch die Gestaltung der Cappella Chigi prägten. Der Paragone mit der Antike spielte in diesem speziellen Fall eine eher untergeordnete Rolle; ${ }^{69}$ dafür kam eine weitere Bezugsgröße hinzu: Raffael, der die von Lorenzetto ausgeführten Skulpturen entworfen hatte.

66 Zur Parallelisierung von Löwengrube und Grab vgl. Hesse (1983), S. 122.

67 Petrucci (1997), S. 181-187; Lavin (1998).

68 Zum Paragone Michelangelo/ Bernini siehe u. a. Thoenes (1983); Preimesberger (1985), S. 6f.; Montanari (1997), S. 58f.; zum Wettstreit mit der Malerei insbesondere Preimesberger (1985). Zur Antikenrezeption sei nur verwiesen auf die zahlreichen exzellenten Katalogbeiträge in: Bernini Scultore, Ausst. Kat. Rom (1998).

69 Fabjan (1999), S. 54, erinnert daran, daß die Physiognomien von Daniel und Habakuk nach dem Vorbild einer antiken Alexanderbüste bzw. des Borgheseschen Kentauren gestaltet sind. Schulze (1989) verteidigt die von Kauffmann zurückgewiesene These, Daniel sei 
Ursprünglich mußte Bernini davon ausgehen, daß seine beiden Figuren neben dem Kapelleneingang aufgestellt werden würden. Der wohl vom Meister selbst initiierte Planwechsel verschaffte seinem Habakuk dann einen viel prominenteren Platz in der Kapelle (rechts neben dem Altar). Gleichzeitig ergab sich aus der neuen Position aber auch die Aufgabe, in einen skulpturalen Wettstreit mit Lorenzettos Jonas (Abb. 5) zu treten, der auf der linken Altarseite das Pendant des Habakuk bilden sollte. Während Daniel bereits vor dem Planwechsel vollendet war, konnte Bernini bei der Gestaltung des Habakuk kreativ auf die neue räumliche Situation reagieren.

1626 sprach Fabio Chigi Lorenzettos Werke in der Kapelle als »due statue bellis[sim]e del Bonaroti« an ${ }^{70}$ - eine Fehleinschätzung, die eine gewisse Rechtfertigung in den kompositionellen Parallelen zwischen Lorenzettos Elias und dem Jesa$j a$ der Sixtinischen Decke finden konnte. ${ }^{71}$ Seit Vasari war allerdings gut bekannt, daß nicht Michelangelo, sondern Raffael für Lorenzettos Statuen den Entwurf geliefert hatte. ${ }^{72}$ Entsprechend korrigierte Fabio die Zuschreibung in seiner ca. 1630 vollendeten Vita Agostino Chigis, brachte aber nochmals die Meinung einiger Zeitgenossen ins Spiel, es handle sich um Werke Michelangelos. ${ }^{73}$ Bernini entwickelte auf dieser Grundlage einen doppelten und dreifachen Paragone: Er berief sich in seiner Konkurrenz mit den 'Skulpturen eines Malers auf Raffaels Erzrivalen Michelangelo, dessen Können dann - wie so oft - als Prüfstein für Berninis eigene bildhauerische Leistung diente. Darüber hinaus rivalisierte er aber nicht nur mit den Großen des cinquecento, sondern versuchte die grundsätzliche Überlegenheit der Skulptur über die Malerei unter Beweis zu stellen.

Wie bereits Raffael bzw. Lorenzetto holte auch Bernini sich Inspiration bei den Propheten der Cappella Sistina: Sein Habakuk imitiert spiegelbildlich Michelangelos Jonas (Abb. 9). ${ }^{74}$ Beide Propheten sitzen massig und breitbeinig da, nackte Füße wippen locker in den Raum hinein. Beide Figuren zeichnen sich durch einen markanten diagonalen Zeigegestus aus, bei dem die dominante zeigende Hand die andere überkreuzt. Und beide treiben die chiastische Torsion auf die Spitze, indem sie sich zwar in die Richtung zurücklehnen, in die sie deuten, gleichzeitig aber in die entgegengesetzte Richtung emporschauen. So wie Jonas entrückt zu Gottvater im Deckenspiegel aufblickt, so staunt Habakuk die himmlische Erscheinung des En-

aus Berninis Laokoon-Studien entwickelt: vgl. Kauffmann (1970), S. 225. Daniels Pose hat insgesamt jedoch wenig mit dem Laokoon zu tun; sie läßt sich vielleicht eher als Rezeption von Renis Heiligem Andreas verstehen: vgl. Pepper (1988), Kat. Nr. 32, tav. 31.

70 Cugnoni (1881), S. 58.

71 Nobis (1979), S. 87.

72 Vasari, hg. v. Milanesi (1906), Bd. 4, S. 369, 578; Landucci (1646), S. 29; Chisiani Sacelli Descriptio, fol. 425r-426v. Nobis (1979), S. 86, sieht keine Hinweise für eine Mitwirkung Raffaels an Lorenzettos Elias; Poeschke (1992), S. 165, hält sie jedoch für sehr wahrscheinlich.

73 Cugnoni (1879), S. 41, 75.

74 Kauffmann (1970), S. 230; vgl. Rohlmann (1995). 
9. Michelangelo, Jonas, ca. 1508-1512. Fresko. Cappella Sistina, Vatikanstaat

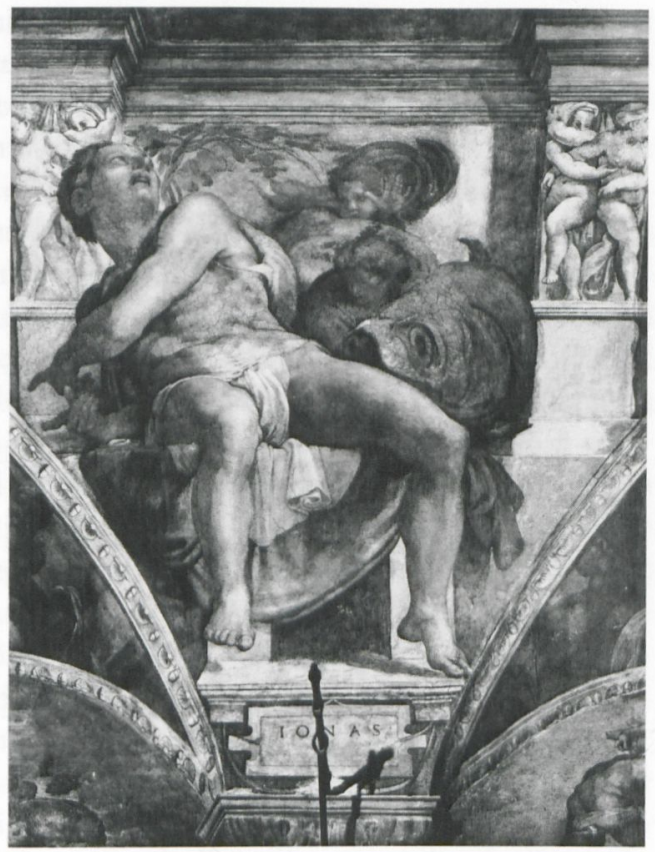

gels an. Daß Habakuk Michelangelos Fresko dabei seitenverkehrt nachahmt, erklärt sich aus Berninis Absicht, die Komposition seiner Skulptur symmetrisch auf Lorenzettos Jonas abzustimmen, der ja Habakuks Pendant bilden sollte. Während Lorenzettos Figur ihr rechtes Bein vorstellt, das linke abwinkelt und sich insgesamt nach rechts wendet (Abb. 5), streckt Habakuk umgekehrt sein linkes Bein vor, stellt das rechte zurück und dreht sich nach links (Abb. 7).

Gerade die symmetrische Koordination der beiden Skulpturen läßt die Unterschiede zwischen ihnen besonders deutlich hervortreten. Wie man es von der 'Skulptur eines Malersi erwarten mag, wirkt der von Raffael entworfene Jonas mehr zweials dreidimensional. Kopf und Rumpf, die Arme und größtenteils auch die Beine der ganz in sich gekehrten Figur befinden sich beinahe in derselben Fläche. ${ }^{75}$ Demgegenüber simuliert Bernini um so energischer räumliche Tiefe und Bewegung. Die Nische ist zu klein für die beiden Personen; Kopf und Arm des Engels stoßen weit in den Kapellenraum hinein. Das fehlende linke Bein des Engels wird von der Phanta-

75 Eine wesentlich dynamischere Zeichnung in Windsor, die manchmal als Raffaels Entwurf für den Jonas angesehen wird, weicht von der Skulptur so stark ab, daß Clayton das Blatt als eine spätere Variante klassifiziert: Clayton (1999), S. 168-171. Poeschke meint hingegen, Lorenzetto habe Raffaels Entwurf verflacht: Poeschke (1992), S. 165. Da Bernini die Zeichnung wohl kaum kannte, dürfte für seinen Paragone nur die Skulptur selbst relevant gewesen sein. 
sie des Betrachters automatisch ergänzt. Man imaginiert es als nach hinten abgewinkelt und mißt der Nische dadurch eine größere (fiktive) Tiefenerstreckung zu. Habakuks Bein ragt dagegen dynamisch in den Kapellenraum vor. Die Diagonale, die von diesem Bein zum weit zurückgelegten Kopf verläuft, erzeugt den Eindruck maximaler räumlicher Tiefe.

Pikanterweise setzte Bernini Raffaels Jonas eine von Michelangelos Jonas inspirierte Figur entgegen und betonte dabei genau die Unterschiede, die auch schon im cinquecento zwischen den beiden Erzrivalen festgestellt worden waren: Auf Raffaels weichen und eher flachen Duktus reagierte er mit virilem rilievo. Michelangelos Jonas galt als Musterbeispiel für sskulpturale Malerei, die die Flächigkeit des Mediums negiert und größtmögliche Plastizität suggeriert. ${ }^{76}$ Bernini begnügte sich aber nicht einfach damit, diese 'gemalte Skulptur in die Dreidimensionalität zurückzuübersetzen und dabei mit der berühmten Virtuosität seiner Oberflächenbehandlung zu glänzen; vielmehr nahm er das vorgegebene Thema zum Anlaß, das schwierige formale Problem der Zweifigurengruppe anzugehen, mit dem auch Michelangelo immer wieder gerungen hatte. ${ }^{77}$ Bravourös verschränkte er die beiden Figuren $\mathrm{zu}$ einer harmonisch ausponderierten Komposition. Obwohl die Protagonisten chiastisch auseinanderstreben (Habakuk lehnt sich nach hinten und weist nach rechts, der Engel beugt sich nach vorne und deutet in die entgegengesetzte Richtung), verbindet Bernini sie einerseits durch ihre spannungsgeladenen Blicke, andererseits durch kompositionelle Vereinheitlichungen, die ein gitterartiges Grundgerüst ergeben: Das rechte Bein des Engels und Habakuks linker Unterschenkel sind vertikal parallelisiert, Habakuks rechter Arm und der linke des Engels hingegen horizontal. Schwungvoll treffen zwei unterschiedliche Bewegungsimpulse zusammen: Am ausgestreckten rechten Arm des Engels und am vorragenden Bein Habakuks entlang gleitet der Blick in die Tiefe und wird von dort durch den linken Arm des Engels und Habakuks parallel laufenden Zeigegestus wieder nach rechts aus der Nische herausgeführt.

Der entscheidende Unterschied zwischen Abb. 5 und Abb. 7 liegt allerdings nicht in der stärker dreidimensionalen, skulpturalen Auffassung von Berninis Gruppe, sondern vielmehr in ihrer narrativen Konzeption. Raffael bzw. Lorenzetto macht nicht anschaulich, daß Jonas gerade dem Wal entstiegen ist. Seine meditative Pose verrät nichts von der Freude des frisch Geretteten, und der viel zu kleine Wal dient nur als Attribut oder quasi als Thron des Propheten, ohne Bestandteil einer Bilderzählung zu sein. Im Gegensatz zu jener isolierten und distanzierten, überzeitlichen Figur fingiert Berninis Gruppe eine dynamische Handlung im Hier und Jetzt. Während Jonas und Elias Sinnbilder für Gerettete sind, thematisiert Bernini das Rettungsgeschehen selbst: Habakuk und der Engel sind soeben in der Löwen-

76 Vasari, hg. v. Milanesi (1906), Bd. 7, S. $185 f$.

77 Die Zweifigurengruppe war quasi ein 'Markenzeichen des Bildhauers Michelangelo, mit dem sich spätere Generationen immer wieder maßen: vgl. Strunck (2000), S. 267275. 
grube eingetroffen, um Daniel Hilfe zu bringen. Noch ganz benommen von seiner wundersamen Flugreise schaut Habakuk staunend den Engel an, der ihn auf Daniel aufmerksam zu machen versucht.

Laut Alberti kann der Künstler durch die Gestaltung einer historia eher als durch einen colossus glänzen. ${ }^{78}$ Bernini scheint sich diesen Satz zu Herzen genommen zu haben. Seine Skulpturen erzählen eine Geschichte, wohingegen der von Raffael entworfene Jonas eher den in sich selbst ruhenden colossus verkörpert. Paradoxerweise arbeitete also der Bildhauer Bernini mit `malerischen` Mitteln, während der Maler Raffael ein höchst statuarisches Werk konzipiert hat. Bernini, nicht Raffael bewältigte die Schwierigkeit, die eigentlich eher der Malerei zugeordnete historia im Medium der Skulptur zu realisieren.

Berninis narrative Auffassung der Geschichte von Daniel und Habakuk ist um so erstaunlicher, als es dafür in vollplastischer Skulptur keine Vorbilder gab. ${ }^{79}$ In Malerei und Graphik war die Begegnung in der Löwengrube jedoch wiederholt dargestellt worden. So waren die Voraussetzungen für einen weiteren Paragone gegeben: Dabei ging es nun nicht mehr nur um den Wettstreit mit Raffael und Michelangelo, sondern ganz grundsätzlich um die unterschiedlichen Möglichkeiten, die Malerei bzw. Skulptur zur Darstellung einer bestimmten Episode besitzen.

Daß Malerei und Graphik sich auf das Zusammentreffen in der Löwengrube konzentrierten, lag in der Natur des Mediums: Wie hätte man denn auf einer begrenzten Fläche beispielsweise den Anfang des Rettungswunders abbilden können, bei dem die beiden Protagonisten sich noch in zwei verschiedenen Ländern befinden? Die zweidimensionale Veranschaulichung jenes Moments liefe darauf hinaus, Daniel und Habakuk winzig klein zu beiden Seiten einer großen Landschaftsszenerie anzuordnen. Eine solche Komposition widerspräche dem Grundprinzip der Historienmalerei, stets den ‘fruchtbaren Moment` zur Darstellung auszuwählen, in dem sich eine groß ins Zentrum gestellte Aktion mit dem Ausblick auf Vergangenheit und Zukunft verbindet. Deswegen schweben in den meisten Gemälden Habakuk und der Engel gerade in die Löwengrube hinein: Sie sind noch im Flug begriffen und erinnern dadurch an ihre wundersame Reise, strecken aber bereits Daniel den Korb hin und machen so die bevorstehende Rettung absehbar.

Die Bildhauerei kann anders mit räumlicher Distanz arbeiten als die Malerei. Die herrschende Forschungsmeinung zur Chigi-Kapelle geht davon aus, daß Bernini sich genau diesen Unterschied zunutze machte, indem er Habakuk und Daniel zu Beginn der Geschichte (noch an verschiedenen Orten) dargestellt habe. Meiner Ansicht nach zeigt die Gestaltung der Figuren jedoch, daß sich beide Protagonisten (der Bild- und Auslegungstradition entsprechend) bereits in der Löwengrube befinden. ${ }^{80}$ Demzufolge bestand Berninis Paragone-Strategie keineswegs

78 Alberti, De Pictura, $\$ 35$ : „Maior enim est ingenii laus in historia quam in colosso «. Alberti (2000), S. 256.

$79 \mathrm{Vgl}$. Anm. 50.

80 Siehe Kapitel 2 des vorliegenden Textes. 
darin, den in Malerei quasi undarstellbaren Moment vor Beginn des Rettungswunders zu präsentieren. Im Gegenteil hielt er sich in seiner Konzeption der Geschichte recht eng an ein malerisches Vorbild (Abb. 8), gestaltete jedoch ein $>$ begehbares Bild ‘, das den Betrachter viel stärker einzubeziehen vermag als jede zweidimensionale Darstellung.

$\mathrm{Daß}$ es Bernini darum ging, den Betrachter zu aktivieren, wird aus mehreren Charakteristika des Ensembles ersichtlich. ${ }^{81}$ Die Kapelle, deren alte Holzschranke durch eine in der Mitte geöffnete Marmorbalustrade ersetzt wurde (Abb. 3), ${ }^{82}$ lädt zum Betreten ein. Die raumgreifenden Gesten Habakuks und des Engels machen neugierig, da ihr Ziel auf den ersten Blick nicht zu erkennen ist. Nur wer sich in die Kapelle begibt versteht, auf wen der Engel zeigt. Daniels Gesicht ist durch seine hoch erhobenen Arme und die Kopfdrehung zunächst jedoch fast ganz dem Blick entzogen. ${ }^{83}$ Der Kapellenbesucher muß um die Figur herumgehen, um den Standort zu finden, der ihm einen Blick auf Daniels Antlitz gewährt. Ebenso ist auch Bewegung erforderlich, um den idealen Betrachterstandpunkt zu suchen, von dem aus sich das gesamte Geschehen erschließt. Dieser Punkt liegt ungefähr in der Kapellenmitte. Wenn der Betrachter dort steht und realisiert, daß sowohl Daniel als auch Habakuk sich in der Löwengrube befinden, dann muß ihm gleichfalls klarwerden, daß er selbst in die fiktive Handlung einbezogen ist. Der Engel weist über seinen Kopf hinweg zu Daniel hinüber. Dessen Rettung scheint sich in demselben raum-zeitlichen Kontinuum zu vollziehen, in dem der Kapellenbesucher sich bewegt.

Der Kunstgriff, Figuren über den Raum hinweg miteinander kommunizieren zu lassen, mag von Verkündigungsgruppen angeregt worden sein; doch sind diese üblicherweise so angebracht, daß der Betrachter sie als Teil einer separaten, von seinem eigenen Bereich abgesonderten Raumsphäre empfindet. Als Vorbild für die Wirkstrategie der Chigi-Kapelle kommt daher eher Berninis David in der Galleria Borghese in Betracht: Die auf einem relativ niedrigen Piedestal postierte Figur lehnt sich dynamisch in die Sphäre des Betrachters vor und fixiert das Publikum angespannt, bereit, im nächsten Augenblick die Wurfschleuder abzufeuern. Der Betrachter befindet sich direkt in der Schußlinie und muß annehmen, daß entweder Goliath hinter ihm steht oder gar er selbst mit Goliath verwechselt wird. ${ }^{84}$

Noch enger als der David scheint mir allerdings Antonio Raggis San Benedetto mit der Konzeption der Chigi-Kapelle zusammenzuhängen. Raggi fertigte das genannte Werk für eine Höhle in Subiaco an, in der der Heilige Benedikt vor seiner Ordensgründung als Eremit gehaust haben soll. Die Höhle war bereits im Mittelalter ein bedeutender Wallfahrtsort und damals mit Malereien geschmückt; später

81 Vgl. Schmitt (1997), S. 238-241.

82 Fabjan (1999), S. 47, 50.

83 Abbildung dieser Seitenansicht bei Schulze (1989), S. 190, Abb. 13.

84 Wittkower (1955), S. 6. 
kamen »fiorami dorati« hinzu. ${ }^{85} \mathrm{Ab}$ Mitte des 17. Jahrhunderts wurde der Ort dann auf ganz andere Art präsentiert: Der nackte Fels wurde freigelegt und Benedikt durch die 1657 aufgestellte Marmorskulptur Raggis vergegenwärtigt. Zu jener heute noch existierenden ,Installation` gehören ferner ein Kreuz und ein Korb aus Marmor. Der Korb erinnert daran, daß Benedikt nur von den Nahrungsmitteln lebte, die ihm ein Freund in die Höhle herabließ ${ }^{86}$ Auch wenn sich die Situation von Benedikt und Daniel in theologischer Hinsicht nicht miteinander vergleichen läßt, so liegt doch die motivische Parallele auf der Hand: In beiden Fällen geht es, banal gesagt, um die Ernährung eines schönen frommen Jünglings in einer lebensfeindlichen Höhle.

In Subiaco war die Höhle als der traditionelle Kultort vorgegeben. Die barocke Umgestaltung, bei der Bernini vielleicht eine Hand im Spiel hatte, ${ }^{87}$ verlieh der Höhle einen neuen devotionalen Fokus, die Heiligenfigur. Mittels Beseitigung aller Ornamentik wurde ein brutaler Kontrast zwischen dem zarten Jüngling und dem harten Felsen erzeugt. Dieser harsche Gegensatz sollte vermutlich die Intensität des religiösen Erlebnisses erhöhen. Der Pilger, der die Höhle betritt, kann sich in die Figur Benedikts hineinprojizieren und begreift schaudernd, wie entbehrungsreich dessen Einsiedlerdasein gewesen sein muß.

Die ästhetische Strategie der Chigi-Kapelle funktioniert ähnlich, aber subtiler. Wegen der bestehenden Bausituation konnte Bernini dort die Höhle nur durch den rohen Felsgrund in Daniels bzw. Habakuks Nischen suggerieren. Der Betrachter, der mit dem Bibeltext und der Bildtradition vertraut ist, kann und soll das Höhlenerlebnis in seiner Imagination komplettieren, um dadurch die existentielle Bedrohung Daniels quasi am eigenen Leib mitzuempfinden.

Natürlich geht es weder in Subiaco noch in der Chigi-Kapelle um eine Totalillusion. Das Kunstwerk soll nach wie vor als Kunstwerk erkennbar bleiben (andernfalls hätte man die Skulpturen farbig gefaßt) ${ }^{88}$ Die ästhetische Grenze wird machtvoll überspielt, nur um auf den inganno den disinganno folgen zu lassen, der dann die Reflexion einleitet. ${ }^{89}$ Die Gestaltung eines Raumes als sbegehbares Bild ‘ ermög-

85 Egidi, Giovannoni, Hermanin (1904), Bd. 1, S. $415 f$.

86 Westin (1978), S. 97-99, 173f. Westins stilistische Datierung (ca. 1665-1670) kann nicht stimmen, da es ein Dokument über die bereits 1657 erfolgte Aufstellung der Skulptur in der Höhle gibt: Iannuccelli (1856), S. 261. Farbaufnahme der Statue bei Giumelli (1982), S. 90, Abb. 81.

87 Iannuccelli (1856), S. 261, betont, der Schöpfer des San Benedetto sei »allievo del cavalier Bernini« gewesen. In der Tat arbeitete Raggi von Mitte 1655 bis Anfang 1658 unter Leitung Berninis an der skulpturalen Dekoration von Santa Maria del Popolo: Westin (1978), S. 146-149. Da sowohl der Hl. Benedikt als auch die Neukonzeption der ChigiKapelle genau in jene Zeit fallen, läßt sich darüber spekulieren, wer hier wen inspirierte. Zum Verhältnis Bernini/ Raggi siehe auch Montagu (1989), S. 140-142.

88 Man denke etwa an die Tradition der Sacri Monti: Perrone (2000).

89 Vgl. Hesse (1983), S. 119-121. 
licht es dem Rezipienten, sich intensiv in die Szene hineinzuversetzen und sich davon emotional besonders stark ansprechen zu lassen; die bewußt ins künstlerische Kalkül einbezogenen Illusionsbrüche sorgen zugleich jedoch für eine Distanzierung, die aus dem Mitfühlen Mitdenken macht, damit der Gläubige auf einer höheren Abstraktionsebene die Vorbildlichkeit von Daniel bzw. Benedikt für die eigene Frömmigkeit erkennt.

Für den Paragone bedeutet das Gesagte, daß Bernini durch das sbegehbare Bild eine spezielle Möglichkeit erschloß, im Wettstreit mit der Malerei zu triumphieren. Indem er durch das Zusammenspiel von Skulptur und Architektur dem Betrachter suggerierte, dieser selbst schmachte mit Daniel in der Löwengrube, schuf er ein dreidimensionales Bild, das den Rezipienten in höchstem Maße affiziert, da es ihn vom Betrachter zum Mitspieler erhebt. Berninis Werk kann somit eine viel stärkere Wirkung entfalten als jede malerische Darstellung der Szene, die durch den Bildrahmen klar von der Welt des Publikums geschieden ist. Aufgrund ihrer speziellen Kommunikationsstruktur übertreffen Berninis Skulpturen nicht nur die statuarischen, den Kontakt mit dem Kapellenbesucher vermeidenden Propheten von Raffael bzw. Lorenzetto, sondern gehen sogar über das hinaus, was Berninis großes Vorbild Michelangelo als Bildhauer geleistet hatte.

\section{Bernini im Wettstreit mit sich selbst: Das Grabmal Alexanders VII.}

Filippo Baldinucci überlieferte die folgende lehrreiche Anekdote aus Berninis Jugend: Wann immer der Knabe seinem Vater Pietro eine Arbeit vorlegte, lobte dieser sie zwar, behauptete aber gleichzeitig, daß Gianlorenzo die nächste Aufgabe sicher nicht ebenso gut bewältigen werde - was den Sohn anstachelte, es um so besser zu machen. Der kluge Vater regte den jungen Künstler auf diese Weise an, sich ständig mit sich selbst zu messen: "tenevalo con se medesimo in continovo cimento «. ${ }^{90}$ Auch in späteren Jahren blieb Gianlorenzo jenem 'paragonalen Schaffensprinzip treu. Immer wieder trat er gewissermaßen mit sich selbst in Wettstreit, um eine bestimmte Idee weiterzuentwickeln und zu perfektionieren. ${ }^{91}$ In diesem Sinne steht die Chigi-Kapelle in einem Paragone mit der Cappella Cornaro und dem Grabmal Alexanders VII. Nachdem es im vorigen Kapitel darum ging, wie Bernini sich in der Chigi-Kapelle mit den Arbeiten anderer Künstler auseinandersetzte, stellt sich nun die Frage, welche Rolle autoreflexives paragonales Denken in Berninis Schaffensprozeß auch werkübergreifend spielte. Dabei treten von der Forschung bisher nicht beachtete konzeptionelle Parallelen zwischen der Cappella Chigi und dem Alexandergrab zutage.

90 Baldinucci (1948), S. 75.

91 Ein Beispiel dafür ist der wiederholte, stets der speziellen räumlichen Situation angepaßte Gebrauch eines auszeichnenden Doppelsäulenmotivs: Strunck (1998), S. 568, 573f. 
Ein Aspekt, der in der Literatur über die Chigi-Kapelle stets zu kurz kommt, ist die Funktion, die ihr Fabio Chigi zudachte. Selbstverständlich wollte er durch die Restaurierung der Kapelle das Prestige seiner Familie heben; ${ }^{92}$ doch sollte der Raum offenbar nicht nur dem Kult lang verstorbener Ahnen dienen, sondern auch in Zukunft als Grablege nutzbar sein. 1627 beschrieb Fabio seinem Onkel Größe und Form der Gruft unter der Kapelle. ${ }^{93}$ Als Innozenz X. den frisch zum Kardinal ernannten Fabio 1652 fragte, welche Titelkirche er sich aussuche, optierte Chigi für Santa Maria del Popolo, denn dort besitze er bereits seine »sepoltura«. Schenkt man seinem Vertrauten und späteren Biographen Sforza Pallavicino Glauben, dann war Fabio damals davon überzeugt, daß er dereinst in Santa Maria del Popolo die letzte Ruhe finden werde. ${ }^{94}$

Bei der Restaurierung der Kapelle in den Jahren 1652-1654 wurde der Zugang zum darunterliegenden Kellerraum vergrößert, was dessen Nutzung als Begräbnisort erleichterte oder überhaupt erst ermöglichte. ${ }^{95}$ Die damals auf der Grabpyramide des Sigismondo Chigi angebrachte Inschrift betonte interessanterweise nicht den genealogischen Bezug zu Kardinal Fabio, sondern zum weltlichen Zweig der Familie: "Sigismundo Chisio [...] Marius et Augustus Chisii Proavo $«{ }^{96}$ Hinter "Marius" und "Augustus" verbergen sich Fabios Brüder, die die familiäre Kontinuität zu sichern hatten. ${ }^{77}$ Wenn man zudem bedenkt, daß Fabio während seines Kardinalats zwei Chigi-Wappen in der Kapelle anbringen ließ, die aber beide nicht mit dem Kardinalshut versehen sind, dann drängt sich der Gedanke auf, der Raum habe nicht nur für Fabio, sondern potentiell für die gesamte Familie als Grabstätte dienen sollen - so wie er später auch tatsächlich genutzt wurde. ${ }^{98}$

92 Fabjan (1999), S. 47-50.

93 Cugnoni (1881), S. 63.

94 Pallavicino (1843), S. 163, überliefert Fabios Antwort auf die Frage Innozenz' X. wie folgt: "Disse il Chigi in S. Maria del Popolo, ove essendo una cappella fondata dai suoi maggiori, aveva la sepoltura, ch'è la vera e stabile casa de' nostri corpi sino al fine del mondo, essendo l'altre più veramente osterie, ove alberghiamo nel pellegrinaggio della vita.« Zur Person Pallavicinos siehe Montanari (1997).

95 Frommel (1974), S. 344, geht davon aus, daß die ursprüngliche Öffnung im Boden mit ca. 80 × $80 \mathrm{~cm}$ zu klein war, um Särge hinabzulassen; die Gruft habe zunächst eher symbolische als praktische Bedeutung besessen.

96 Abbildung der beiden Pyramiden mitsamt Inschrift bei Angelini (1998), S. 138. Die Chisiani Sacelli Descriptio (ca. 1655) unterstreicht explizit, daß Fabio seine Brüder in den Vordergrund stellte: "non suo, sed Marij, et Augusti fratrum nomine« (fol. 29r).

97 Zur Genealogie vgl. Weber (1999), Bd, 29/1, S. LXXVIIf., 241. Augustos Sohn Agostino sowie Marios Sohn Flavio traten 1658 als weltlicher bzw. geistlicher Nepot Alexanders VII. an den Querhausaltären in Santa Maria del Popolo prominent in Erscheinung: vgl. Ackermann (1996), S. 416, 418.

981660 errichtete Mario Chigi eine neue Kaplanei für die Chigi-Kapelle: Ackermann (1996), S. 389. Im November bzw. Dezember 1667 wurden Mario und seine Frau in Santa Maria del Popolo beigesetzt: Bentivoglio, Valtieri (1976), S. 229. 


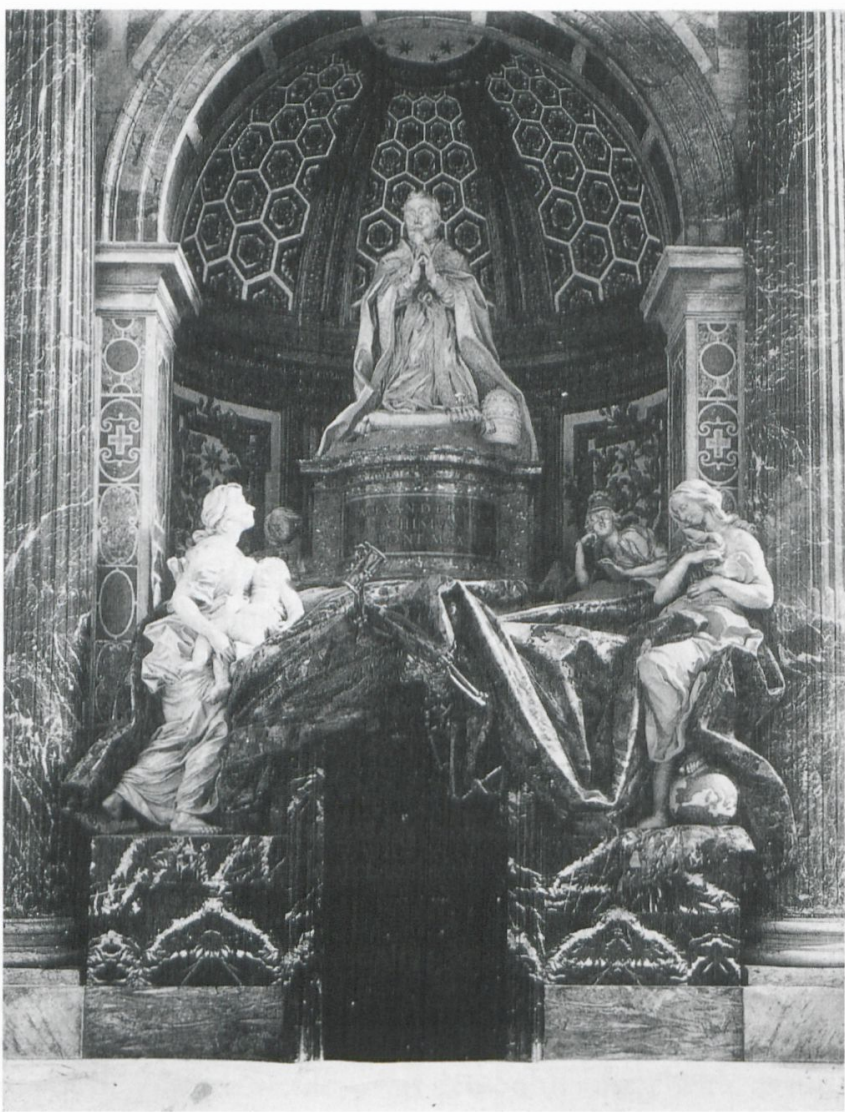

10. Gianlorenzo Bernini und Werkstatt, Grabmal Alexanders VII., 1671-1678. St. Peter, Vatikanstaat

Schon gleich nach seiner Erhebung zum Papst umgab sich Fabio bzw. Alexander VII. mit Symbolen der Vergänglichkeit - einem Sarg, einem Totenschädel. ${ }^{99}$ Wenig später berichtete ein Avviso: "già fece fare la cassa per riponervi il Suo Cadavere, hora dice si facci fare il disegno della Sua Sepoltura, et egli med[esi]mo comporrà l'Iscritt[io]ne Sepulcrale. « ${ }^{100}$ Ein Dokument vom April 1656 belegt, daß Alexander VII. im Petersdom beigesetzt werden wollte. ${ }^{101}$ Die Chigi-Kapelle, die der Kardinal sich als Grablege hergerichtet hatte, war für einen Papst offenbar nicht repräsentativ genug. Im August 1656 vergab Alexander den Auftrag für sein neues Sepulkral-

99 Zollikofer (1994), S. 116.

100 Avviso vom 28. August 1655, zit. nach Petrucci (1997), S. 181.

101 Zollikofer (1994), S. 11, 118. 
monument an Bernini, doch wurde es letztlich erst in den Jahren 1671-1678 ausgeführt. 1667 wurde Alexanders Körper zunächst provisorisch in St. Peter bestattet, ${ }^{102}$ während immerhin sein Herz in Santa Maria del Popolo die letzte Ruhe fand. ${ }^{103}$

Da Chigi und Bernini die Kapelle in Santa Maria del Popolo ursprünglich als Grabstätte für den Kardinal und die Seinen geplant hatten, war es nur logisch, daß ihre Überlegungen zum Alexandergrab von der Cappella Chigi ausgingen, zumal jene Planungen und die Arbeiten an der Chigi-Kapelle zeitweilig parallel nebeneinander herliefen. ${ }^{104}$ Das schließlich realisierte Monument (Abb. 10) hat auf den ersten Blick allerdings wenig mit den Gräbern in Santa Maria del Popolo gemein. Bei genauerem Hinsehen wird jedoch ein autoreflexiver Paragone erkennbar, durch den Bernini die Konzeption der Cornaro- und Chigi-Kapellen weiterentwickelte.

In der Cappella Cornaro (1647-1651) gestaltete Bernini die Durchdringung verschiedener Realitätsebenen. Er konfrontierte den Kapellenbesucher mit Wesen aus den unterschiedlichsten Sphären: Von oben scheinen himmlische Heerscharen in den Raum einzubrechen, ja ein Engel fliegt gar bis zum Altar hinab, um dort die ihm auf Wolken entgegenschwebende Heilige Theresa mit Gottesliebe zu entflammen - eine Tat, die die verstorbenen Vorfahren des Kapellengründers beobachten und so lebhaft kommentieren, als seien sie noch am Leben, während unten aus der Gruft zwei Skelette zur himmlischen Vision emporstreben. ${ }^{105}$

Fabio Chigi besichtigte dieses unerhörte Kunstmirakel am 7. Februar 1653 und ging anschließend direkt in Berninis Werkstatt, wohl um das Gesehene zu diskutieren. ${ }^{106}$ Dabei kam man offenbar überein, den in der Cappella Cornaro so meisterhaft visualisierten Zusammenhang von Unter- und Oberwelt, Tod und ewigem Leben auch für die Chigi-Kapelle fruchtbar zu machen. Die opus-sectile-Darstellung eines Skeletts, die Bernini für Fabio Chigi entwarf, findet ihr direktes Vorbild in der Cappella Cornaro ${ }^{107}$ und wurde auch von demselben Steinmetzen Gabriele

102 Zollikofer (1994), S. 12; Pinelli (2000), S. 593 f.

103 Bentivoglio, Valtieri (1976), S. 228. 1667 ging sogar das Gerücht um, daß das Grabmonument des Papstes in Santa Maria del Popolo errichtet werden solle: Zollikofer (1994), S. 121 (Notizen des Carlo Cartari, 24. und 25. Mai 1667).

104 Wie aus dem Tagebuch Alexanders VII. hervorgeht, waren die Planungen für sein Grab zwischen 1655 und 1660 in vollem Gange, d. h. vor Fertigstellung der Kapelle in Santa Maria del Popolo. Die definitive Form des Grabmonuments soll noch zu Alexanders Lebzeiten festgelegt worden sein: Zollikofer (1994), S. 11, 120f., 123f. Die Problematik der Chronologie beleuchtet Montanari, in: Pinelli (2000), S. 594-596.

105 Für eine differenzierte Analyse des hochkomplexen Kunstwerks, die hier natürlich nicht geleistet werden kann, sei auf Lavin (1980) und Preimesberger (1986) verwiesen.

106 Tagebucheintrag, abgedruckt bei Petrucci (1998), S. 183.

107 Samek Ludovici weist übrigens darauf hin, daß auch der Habakuk begleitende Engel in der Chigi-Kapelle von der Cappella Cornaro inspiriert ist: Baldinucci (1948), S. 231, Anm. 87. 
Renzi ausgeführt, der die Gerippe für das Paviment der Cornaro-Kapelle geschaffen hatte. ${ }^{108}$

Bernini kopierte seine eigene Idee allerdings nicht einfach, sondern paßte sie kreativ der Situation in der Chigi-Kapelle an. Während in der Cappella Cornaro zwei relativ kleine opus-sectile-Medaillons mit halbfigurigen Gerippen in den Fußboden seitlich vor dem Altar eingelassen wurden, ${ }^{109}$ plazierte Bernini im Zentrum der Cappella Chigi nur ein wappentragendes geflügeltes Skelett auf der großen runden Steinplatte, die die Gruft verschließt (Abb. 2). Die Form dieser Steinplatte nimmt die kreisrunde Form des Kuppel-Opaions auf - eine optische Parallelisierung, der zuliebe extra der bisherige Zugang zur Gruft in die Raummitte verlegt wurde. ${ }^{110}$ Das Skelett, das das Chigi-Wappen aus der Gruft emporträgt, wurde so in eine direkte visuelle Beziehung zu dem segnenden Gottvater gesetzt, der durch die fingierte runde Kuppelöffnung in die Kapelle hinabschaut. Unter- und überirdische Welt kommunizieren mit der Welt des Kapellenbesuchers; das Skelett fliegt aus der Gruft durch die Kapelle zu Gott hinauf und veranschaulicht so die Auferstehungshoffnung. Während in der Cappella Cornaro die Gerippe dargestellt sind, die sich am jüngsten Tag aus ihren Gräbern erheben, ${ }^{111}$ befinden sich die ChigiAhnen (vertreten durch ihr Wappen) bereits auf dem Weg zum ewigen Leben: "Mors aD CaeLos [Iter]«.

Die Skelette im Fußboden der Cornaro- und Chigi-Kapelle verweisen auf die Gruft, die unter dem Kapellenbesucher liegt. Berninis Neukonzeption der Cappella Chigi während des Pontifikats Alexanders VII. brachte jedoch die bisherige Gliederung des Raumes in drei horizontale Zonen durcheinander. Er versetzte nun den Kirchgänger quasi mitten in die Gruft hinein, um die Betrachteransprache zu intensivieren. ${ }^{112}$ Die klare Abgrenzung zwischen Erdenwelt und Unterwelt verwischte sich: Der Kapellenbesucher steht in der Löwengrube und damit symbolisch im Grab. Diese Idee entwickelte Bernini schließlich in seinem Sepulkralmonument für Alexander VII. weiter.

Auch das Alexandergrab (Abb. 10) gliedert sich in drei horizontal übereinandergestaffelte Zonen. Wie in der Chigi-Kapelle überwölbt eine ,Himmels-Kuppel ‘ das Monument. ${ }^{113}$ Darunter erstreckt sich eine sirdische Zone, die der Papst und seine

108 Petrucci (1998), S. 188. Petruccis Ansicht, das Skelett für die Cappella Chigi sei schon 1652 entworfen worden, überzeugt nicht, da mit dem 1652 in Chigis Tagebuch erwähnten »arme« bzw. "scudo" das am Kapelleneingang angebrachte Wappen gemeint sein dürfte (siehe oben, Anm. 20 und 35).

109 Lavin (1980), Bd. 2, Abb. 192.

110 Vgl. Frommel (1974).

111 Lavin (1980), Bd. 1, S. 134-137.

112 Siehe oben, Kapitel 2 und 3.

113 Wegen ihrer Kassettierung wird diese Kuppel gemeinhin mit den Kuppeln im Pantheon, Sant'Andrea al Quirinale, Santa Maria dell'Assunzione in Ariccia und San Tommaso da Villanova in Verbindung gebracht: Bernstock (1988), S. 185; Zollikofer (1994), S. 58-62; Marder (1998), S. 316. Die Gliederung der Kuppel durch Rippen, der zen- 
Tugenden einnehmen. Zuunterst fliegt der Tod aus der Tür der `Grabkammer ‘. ${ }^{114}$ Wie in der Chigi-Kapelle wird der Betrachter direkt mit dem Tod konfrontiert. Der Tod ist nicht mehr dem Fußboden und der darunterliegenden Gruft zugeordnet, sondern kommt dem Betrachter quasi 'von gleich zu gleich ‘ aus einer ebenerdigen Grabkammer entgegen. Da es sich bei der Pforte der scheinbaren Grabkammer um eine häufig benutzte Durchgangstür handelte und noch handelt, ${ }^{115}$ kann man es mit Schaudern erleben, daß jemand in das , Grab hineingeht. Die Omnipräsenz des Todes, der mitten ins Leben hineindrängt, wird dadurch ebenso wie in der ,Löwengrube von Santa Maria del Popolo unmittelbar anschaulich und ermahnt den Gläubigen dazu, sein Leben im Hinblick auf Tod und Fegefeuer moralisch korrekt zu gestalten - wie es sowohl Alexander VII. als auch Bernini durch ihre eigene religiöse Praxis propagierten. ${ }^{116}$

Die Botschaft des Alexandergrabes lautet, daß der Papst durch seine Tugenden über den Tod triumphiere. ${ }^{117}$ Der Tod öffnet die Tür zum Jenseits, das letzten Endes seinen Schrecken verliert. Der sternenbesäte Himmel erwartet den Tugendsamen; sein geflügeltes Wappen hat sich bereits hoch über den Nischenbogen erhoben - eine Variation auf das vom Tod emporgetragene Wappen in der Chigi-Kapelle. Auch über dem Alexandergrab könnte das Motto stehen: "Mors aD CaeLos Iter«.

\section{Zusammenfassung}

„Oh veramente felice età nostra! oh beati artefici! « jubelte Giorgio Vasari angesichts von Michelangelos Jonas in der Sixtinischen Kapelle. ${ }^{118}$ Ungefähr ein Jahrhundert später zitierte Bernini genau jene viel bewunderte Kunstfigur, um den von Raffael entworfenen Jonas der Cappella Chigi zu übertreffen: Gegen die Skulptur des Malers rief er das Gemälde des Bildhauers auf den Plan (Abb. 5, 9). Bernini übersetzte

trale Okulus und vor allem die Sterne auf blauem Grund erinnern aber auch an die Chigi-Kapelle, deren Kuppel übrigens ebenfalls teilweise kassettiert ist.

114 Vgl. Bialostocki (1973); Bernstock (1988), S. 176, 178f. Mit der Verbindung von Grab und Tür hatte Bernini bereits 1654 in einem Projekt für das Grabmonument Innozenz' X. experimentiert, ohne daß dort allerdings die Tür als Pforte zur Grabkammer konnotiert war: vgl. Gampp (1997/98), speziell S. 486-489. Die Kombination von Tür und Tod (ohne überfangende sHimmels-Kuppel) findet sich in einem Entwurf Berninis für ein Dogengrab, dessen Datierung in die frühen 1650er Jahre auf einer sehr hypothetischen Argumentation beruht: Blunt (1967), S. 231.

115 Bernstock (1988), S. 170, 172. Laut Zollikofer hätte das Grab zunächst in einer anderen Nische untergebracht werden sollen; die Tür war jedoch von Anfang an Teil des Projekts: Zollikofer (1994), S. 24, 30, 38, 66.

116 Petrucci (1997), S. 181-187; Lavin (1998).

117 Bernstock (1988), S. 176ff.; Montanari, in: Pinelli (2000), S. 598.

118 Vasari, hg. v. Milanesi (1906), Bd. 7, S. 186. 
die für ihre plastischen Qualitäten gepriesene Malerei Michelangelos in Skulptur zurück und trieb dabei das chiastische Bewegungsmotiv des sixtinischen Jonas auf die Spitze, indem er es zu einer Zweifigurengruppe weiterentwickelte. Durch das genau ausponderierte Spannungsverhältnis zwischen Habakuk und dem Engel (Abb. 7) bewältigte Bernini souverän die von seinem großen Vorbild Michelangelo vielfach umkreiste difficoltà, zwei Skulpturen zu einer überzeugenden Komposition zu verbinden. Im Vergleich zu ihrem raffaelesken Gegenpart in der Chigi-Kapelle brilliert die Habakuk-Gruppe durch virtuose Oberflächenbehandlung, kraftvolle Tiefenerschließung, kompositionelle und emotionale Dynamik - doch als die eigentliche Trumpfkarte in diesem Paragone setzte Bernini ein innovatives erzählerisches Konzept ein, durch das er den Betrachter in besonders intensiver Weise anzusprechen suchte.

Wie eine bislang unbeachtete Kapellenbeschreibung und eine Zeichnung (Abb. 3) beweisen, waren Jonas und Elias während der Restaurierungsarbeiten der Jahre 1652-1654 zu beiden Seiten des Altares plaziert worden. Erst nach seiner Papstwahl faßte Alexander VII. 1655 den Entschluß, für die beiden verbleibenden Nischen Skulpturen bei Bernini in Auftrag zu geben. Während sich Daniel spätestens 1656 in Arbeit befand, scheint Bernini die definitive Form der Habakuk-Gruppe nicht früher als 1658 entwickelt zu haben. Erst in jenem Jahr fiel wohl die Entscheidung, das bisherige Aufstellungskonzept zu verändern, um Daniel und Haba$k u k$ diagonal über den Raum hinweg miteinander kommunizieren zu lassen.

Die Abweichung von der ursprünglich vorgesehenen Statuenverteilung reflektierte ein neues inhaltliches Programm. Während die Propheten zuvor als isolierte Figuren gedacht waren, gestaltete Bernini Daniel und Habakuk nun als miteinander interagierendes Paar. Habakuk und der Engel scheinen soeben bei Daniel eingetroffen zu sein; dieser dankt Gott für seine Rettung. Der einheitliche Felsgrund in beiden Statuennischen suggeriert, daß alle Protagonisten sich an demselben Ort befinden - und der Betrachter steht mitten zwischen den Figuren, also genau in der Löwengrube.

Während die 1652-1654 durchgeführte Kapellenrestaurierung die von Raffael angelegte Gliederung des Baus in drei horizontale Zonen (Unterwelt bzw. Gruft, Erdenwelt, Himmel bzw. Kuppel) beibehalten hatte, durchbrach Bernini jenes Schema in der letzten Phase der Programmgenese: Da die Löwengrube traditionell als Sinnbild für das Grab verstanden wurde, versetzte Bernini den Kapellenbesucher quasi mitten in die Welt des Todes hinein. Diese Wirkstrategie, die möglicherweise durch die damalige Neuinszenierung der Grotte des Hl. Benedikt in Subiaco angeregt worden war, sollte es dem Gläubigen ermöglichen, sich ganz unmittelbar mit Daniel zu identifizieren, seine existentielle Bedrohung und die Freude über Gottes Hilfe mitzufühlen. Durch die Intensivierung des religiösen Erlebnisses bewies Bernini die Überlegenheit der Skulptur im Paragone mit der Malerei: Er gestaltete gewissermaßen ein ‘begehbares Bild`, das den Kapellenbesucher zum Mitspieler machte und ihn folglich viel stärker zu bewegen vermochte als jede zweidimensionale Darstellung des Geschehens. Gleichzeitig übertraf Bernini auf diese Weise die sSkulpturen eines Malers`, denn während Raffael Jonas und Elias als in 
sich ruhende, sstatuarischer Objekte konzipiert hatte, faßte Bernini sein Thema >malerisch «-narrativ auf und schlug so den Maler Raffael gewissermaßen mit dessen eigenen Waffen.

Da Fabio Chigi die Kapelle in Santa Maria del Popolo zunächst als Grablege für sich und die Seinen herrichten ließ, mag es für Bernini nur folgerichtig gewesen sein, die in der Chigi-Kapelle verwirklichten Ideen für das Prunkgrab in St. Peter 'wiederzuverwenden', das er nach Fabios Papstwahl zu entwerfen begann. Auch dieses Monument gliedert sich in drei horizontale Zonen, und wie in der Chigi-Kapelle wird der Betrachter direkt mit der Welt des Todes konfrontiert (Abb. 10). Der neuartige Gedanke, die Trennung von Gruft und Erdenwelt scheinbar aufzuheben und den Kapellenbesucher in die `Höhle des Todes` zu schicken, begegnet am Alexandergrab in noch prononcierterer Form, indem ein Skelett dem Betrachter aus einer tatsächlich benutzbaren Tür entgegenfliegt. In einem autoreflexiven Paragone entwickelte Bernini so das Konzept der Chigi-Kapelle weiter - auch im solchermaßen stetigen Wettstreit mit sich selbst seinem Vorbild Michelangelo nicht unähnlich: »o fusse pure che il giudizio di quello uomo fussi tanto grande, che non si contentava mai di cosa che e' facessi ${ }^{119}$

\section{Literatur:}

Felix Ackermann, „Berninis Umgestaltung des Innenraumes von S. Maria del Popolo unter Alexander VII. (1655-1659)«, in: Römisches Jahrbuch der Bibliotheca Hertziana 31, 1996, S. 369-426.

Jacopo Alberici, Compendio delle Grandezze dell'illustre, et devotissima Chiesa di Santa Maria del Populo di Roma, Rom 1600.

Leon Battista Alberti, Das Standbild. Die Malkunst. Grundlagen der Malerei, hg. v. Oskar Bätschmann, Christoph Schäublin, Kristine Patz, Darmstadt 2000.

Alessandro Angelini, Gian Lorenzo Bernini e i Chigi tra Roma e Siena, con un saggio di Tomaso Montanari [...], Siena 1998.

-, Bernini, Mailand 1999.

Daniel Arasse, L'annonciation italienne. Une histoire de perspective, Paris 1999.

Charles Avery, Bernini, München 1998.

Filippo Baldinucci, Vita di Gian Lorenzo Bernini, hg. v. Sergio Samek Ludovici, Mailand 1948.

Ernst Benkard, Giovanni Lorenzo Bernini, Frankfurt a. M. 1926.

Enzo Bentivoglio, "La Cappella Chigi. L'immagine ritrovata del secondo pannello bronzeo del monumento di Agostino, gli inediti precendenti artistici del mosaicista Luigi (da Pace) veneziano ed altre considerazioni«, in: Raffaello a Roma. Il convegno del 1983 (Kongreßakten Rom 1983), Rom 1986, S. 309-314.

-, "La Cappella Chigi«, in: Christoph Luitpold Frommel, Stefano Ray, Manfredo Tafuri (Hg.), Raffaello Architetto, Mailand 1984, S. 125-142.

-, Simonetta Valtieri, Santa Maria del Popolo, Rom 1976.

119 Vasari, hg. v. Milanesi (1906), Bd. 7, S. 243. 
Domenico Bernino [Bernini], Vita del Cavalier Gio. Lorenzo Bernino, Rom 1713, Reprint Perugia 1999.

Bernini Scultore. La nascita del Barocco in Casa Borghese (Ausst. Kat. Rom 1998), hg. v. Anna Coliva, Sebastian Schütze, Rom 1998.

Judith Bernstock, "Bernini's Tomb of Alexander VII «, in: Saggi e memorie di storia dell'arte 16, 1988, S. 167-190, 363-373.

Jan Białostocki, "The Door of Death. Survival of a Classical Motif in Sepulchral Art«, in: Jahrbuch der Hamburger Kunstsammlungen 18, 1973, S. 7-32.

Biblia Sacra Vulgatae Editionis Sixti V Pont. Max. Iussu Recognita et Clementis VIII Auctoritate Edita, Città del Vaticano 1965.

Anthony Blunt, "Two Drawings for Sepulchral Monuments by Bernini«, in: Essays in the History of Art presented to Rudolf Wittkower, hg. v. Douglas Fraser, Howard Hibbard, Milton J. Lewine, London 1967, S. 230-232.

Nicolas Bock, Kunst am Hofe der Anjou-Durazzo. Der Bildhauer Antonio Baboccio (1351ca. 1423), München, Berlin 2001.

Max von Boehn, Lorenzo Bernini, seine Zeit, sein Leben, sein Werk, Bielefeld, Leipzig 1912.

Antonio Bosio, Roma Sotterranea, Rom 1650.

Paul Fréart de Chantelou, Journal de voyage du Cavalier Bernin en France. Édition de Milovan Stanic, Paris 2001.

Chisiani Sacelli Descriptio Addenda Operi: Roma Vetus, et Recens, Auctore Alexandro Donato Senensi (Biblioteca Apostolica Vaticana, Mss. Chigiani N.III.78, fol. 421r-430v nach moderner Zählung), ca. 1655.

Etienne Choppy, L’Annonciation, Marseille 1991.

Martin Clayton, Raphael and his Circle. Drawings from Windsor Castle, London 1999.

Giuseppe Cugnoni, »Agostino Chigi il Magnifico«, in: Archivio della Società Romana di Storia Patria 2, 1879, S. 37-83.

-, "Note al Commentario di Alessandro VII sulla Vita di Agostino Chigi«, in: Archivio della Società Romana di Storia Patria 3, 1880, S. 213-232, 291-305, 422-448.

-, "Note al Commentario di Alessandro VII sulla Vita di Agostino Chigi«, in: Archivio della Società Romana di Storia Patria 4, 1881, S. 56-75.

-, "Appendice al Commento della Vita di Agostino Chigi il Magnifico", in: Archivio della Società Romana di Storia Patria 6, 1883, S. 497-539.

Maarten Delbeke, » A Note on the Immaculist Patronage of Alexander VII: Chigi and the Pilgrimage Church of Scherpenheuvel in the Low Countries«, in: Bulletin de l'Instiut Historique Belge de Rome, 71, 2001, S. 167-200.

Alessandro Donati, Roma vetus ac recens utriusque aedificiis ad eruditam cognitionem expositis, Romae 1665.

P. Egidi, G. Giovannoni, F. Hermanin, I monasteri di Subiaco, Rom 1904.

Barbara Fabjan, "Fabio Chigi, la Cappella di Santa Maria del Popolo e il `Cav. Bernino«, in: Gian Lorenzo Bernini. Regista del Barocco. I restauri, hg. v. Claudio Strinati, Maria Grazia Bernardini, Mailand 1999, S. 47-56.

Oreste Ferrari, Serenita Papaldo, Le sculture del Seicento a Roma, Rom 1999.

Stefano Filippi, "La Cappella Chigi«, in: Fabbriche romane del primo '500. Cinque secoli di restauri (Ausst. Kat. Rom, Bari 1984), Rom 1984, S. 111-137.

Oskar Fischel, Raphael, London 1948.

Stanislao Fraschetti, Il Bernini. La sua vita, le sue opere, il suo tempo, Mailand 1900.

Christoph Luitpold Frommel, "Das Hypogäum Raffaels unter der Chigikapelle in S. Maria del Popolo zu Rom«, in: Kunstchronik 27, 1974, S. 344-348.

Axel Christoph Gampp, "Onde il Bernino è restato il factotum. Ein unbekannter Entwurf 
Berninis für ein Papstgrabmal im Kontext der Baugeschichte von Sant'Agnese«, in: Römisches Jahrbuch der Bibliotheca Hertziana 32, 1997/98, S. 479-506.

Claudio Giumelli (Hg.), I monasteri benedettini di Subiaco, Cinisello Balsamo 1982.

Domenico Gnoli, "La sepoltura d'Agostino Chigi nella chiesa di S. Maria del Popolo in Roma", in: Archivio Storico dell'Arte 2, 1889, S. 317-326.

Vincenzo Golzio, „Documenti berniniani«, in: Archivi d'Italia 1, 1933/34, S. 138-145.

Dieter Graf, Erich Schleier, "Some unknown works by Guglielmo Cortese«, in: The Burlington Magazine 115, 1973, S. 794-801.

Ingo Herklotz, "Fabriche di Roma nel 1651, 1652, 1653. Kunst- und baugeschichtliche Nachrichten aus dem Diario des Giuseppe Gualdi«, in: Analecta Romana Instituti Danici 29 (2003), S. 193-233.

Michael Hesse, "Berninis Umgestaltung der Chigi-Kapelle an S. Maria del Popolo in Rom", in: Pantheon 41, 1983, S. 109-126.

Howard Hibbard, Bernini, Harmondsworth 1965.

Michael Hirst, "The Chigi Chapel in S. Maria della Pace«, in: Journal of the Warburg and Courtauld Institutes 24, 1961, S. 161-185.

Gregorio Iannuccelli, Memorie di Subiaco e sua Badia, Genua 1856.

Nicola Ivanoff, „Un contributo a Sebastiano Mazzoni«, in: Arte veneta 1, 1947, S. $42-45$.

J. Richard Judson, Dirck Barendsz. 1534-1592, Amsterdam 1970.

Hans Kauffmann, Giovanni Lorenzo Bernini. Die figürlichen Kompositionen, Berlin 1970.

Richard Krautheimer, Roger B. S. Jones, "The Diary of Alexander VII. Notes on Art, Artists and Buildings", in: Römisches Jahrbuch für Kunstgeschichte 15, 1975, S. 199235.

Hanno-Walter Kruft, "Gabriele di Battista, alias da Como: problemi sull'identità e le opere di uno scultore del Rinascimento in Sicilia", in: antichità viva 15/6, 1976, S. 18-38.

-, Antonello Gagini und seine Söhne, München 1980.

-, Francesco Laurana. Ein Bildhauer der Frührenaissance, München 1995.

Rudolf Kuhn, "Gian Paolo Oliva und Gian Lorenzo Bernini«, in: Rudolf Kuhn, Gesammelte Beiträge zur Auslegung seiner Skulpturen, Frankfurt a. M. u. a. 1993, S. 163-166.

Ambrogio Landucci, Origine Del Tempio Dedicato in Roma alla Vergine Madre di Dio Maria Presso alla Porta Flaminia, detto hoggi del Popolo, Rom 1646.

Irving Lavin, Bernini and the unity of the visual arts, New York, London 1980.

-, Bernini e il Salvatore. La »buona morte« nella Roma del Seicento, Rom 1998.

Tod Marder, Gian Lorenzo Bernini, Mailand 1998.

Egidio Martini, „Paris Bordon e la ritrovata pala di Santa Marina", in: Arte illustrata 1/7-12, 1968, S. 22-27.

David McTavish, Giuseppe Porta Called Giuseppe Salviati, New York, London 1981.

Priscilla Grazioli Medici, "Sixteenth- and Seventeenth-Century Marble Incrustations in Rome: The Chigi Chapel«, in: The Princeton Raphael Symposium. Science in the Service of Art History, hg. v. John Shearman, Marcia B. Hall, Princeton 1990, S. 93-98.

William Melczer, "Raffaello e il neoplatonismo. La Cappella Chigi di Santa Maria del Popolo«, in: Raffaello e l'Europa. Atti del IV Corso Internazionale di Alta Cultura (Kongreßakten Rom 1990), hg. v. Marcello Fagiolo, Maria Luisa Madonna, Rom 1990, S. 153175.

Jörg Martin Merz, Pietro da Cortona. Der Aufstieg zum führenden Maler im barocken Rom, Tübingen 1991.

Wilhelm Messerer, "Zu Berninis Daniel und Habakuk«, in: Festschrift Engelbert Kirchbaum S. J. (Römische Quartalschrift 57, 1962), S. 292-296.

Benedetto Millino [Mellini], Saggio Della Roma Descritta Da Benedetto Millino. Piazza e Chiesa del Popolo (Biblioteca Apostolica Vaticana, Mss. Chigiani.O.VII.141), ca. 1656. 
Jennifer Montagu, Roman Baroque Sculpture. The industry of art, London 1989.

Tomaso Montanari, „Gian Lorenzo Bernini e Sforza Pallavicino«, in: Prospettiva 87/88, 1997, S. 42-68.

-, "Bernini e Cristina di Svezia. Alle origini della storiografia berniniana«, in: Angelini (1998), S. 328-477.

Giovanni Morello, "Bernini e i lavori a S. Pietro nel diario' di Alessandro VII «, in: Bernini in Vaticano (Ausst. Kat. Città del Vaticano 1981), Rom 1981, S. 321-340.

-, "I rapporti tra Alessandro VII e Gian Lorenzo Bernini negli autografi del papa (con disegni inediti) «, in: Documentary Culture. Florence and Rome from Grand-Duke Ferdinand I to Pope Alexander VII (Kongreßakten Florenz 1990), hg. v. Elizabeth Cropper, Giovanna Perini, Francesco Solinas (Villa Spelman Colloquia, 3), Bologna 1992, S. 185207.

Antonio Munoz, G. L. Bernini. Architetto e Decoratore, Rom 1925.

Elvira Stefania Natoli, "Contributi a Pietro Novelli«, in: Commentari 14, 1963, S. 171-182.

Norbert Walther Nobis, Lorenzetto als Bildhauer, Diss. Bonn 1979.

Giovanni Paolo Oliva, Prediche dette nel Palazzo Apostolico. Tomo secondo, o. O. o. J. [Rom 1664].

-, Prediche dette nel Palazzo Apostolico, Rom 1674.

Steven Ostrow, „Daniel. Rome, S. Maria del Popolo, Chigi Chapel«, in: Drawings by Gianlorenzo Bernini from the Museum der Bildenden Künste Leipzig, German Democratic Republic (Ausst. Kat. Princeton 1981), hg. v. Irving Lavin, Princeton 1981, S. 164-173. Sforza Pallavicino, Vita di Alessandro VII Sommo Pontefice Libri cinque, Mailand 1843.

Stephen Pepper, Guido Reni. L'opera completa, Novara 1988.

Adriano Peroni (Hg.), Il Duomo di Pisa (Mirabilia Italiae, 3), Modena 1995.

Stefania Stefani Perrone, "Giovanni d'Enrico«, in: Tanzio da Varallo. Realismo fervore e contemplazione in un pittore del Seicento (Ausst. Kat Mailand 2000), Mailand 2000, S. 197-207.

Francesco Petrucci, „Gian Lorenzo Bernini per casa Chigi: precisazioni e nuove attribuzioni«, in: Storia dell'arte 90, 1997, S. 176-200.

-, "Bernini, Algardi, Cortona ed altri artisti nel diario di Fabio Chigi cardinale (16521655)«, in: Rivista dell'Istituto Nazionale d'Archeologia e Storia dell'Arte 53, 1998, S. 169-196.

-, Kat. Nr. 73-78, in: Maria Grazia Bernardini, Maurizio Fagiolo dell'Arco (Hg.), Gian Lorenzo Bernini. Regista del Barocco (Ausst. Kat. Rom 1999), Mailand 1999, S. 355-358.

Antonio Pinelli (Hg.), La Basilica di San Pietro in Vaticano (Mirabilia Italiae, 10), Modena 2000 .

-, "La cappella delle tombe scambiate. Novità sulla Cappella Chigi in Santa Maria del Popolo", in: Catherine Monbeig Goguel, Philippe Costamagna, Michel Hochmann (Hg.), Francesco Salviati et la bella maniera (Kongreßakten Rom, Paris 1998), Rom 2001, S. 253-285.

Joachim Poeschke, Die Skulptur der Renaissance in Italien. Bd. 1: Donatello und seine Zeit, München 1990.

-, Die Skulptur der Renaissance in Italien. Bd. 2: Michelangelo und seine Zeit, München 1992.

Friedrich Pollak, Lorenzo Bernini. Eine Studie, Stuttgart 1909.

John Pope-Hennessy, Donatello Sculptor, New York, London, Paris 1993.

Paolo Portoghesi (Hg.), Caprarola, Rom 1996.

Rudolf Preimesberger, "Themes from Art Theory in the Early Works of Bernini«, in: Gianlorenzo Bernini. New Aspects of His Art and Thought, hg. v. Irving Lavin, University Park, London 1985, S. 1-24. 
-, "Berninis Cappella Cornaro. Eine Bild-Wort-Synthese des siebzehnten Jahrhunderts? Zu Irving Lavins Bernini-Buch", in: Zeitschrift für Kunstgeschichte 49, 1986, S. 190219.

Anthony Radcliffe, Malcolm Baker, Michael Maek-Gérard, The Thyssen-Bornemisza Collection. Renaissance and later sculpture with works of art in bronze, London 1992.

Marcel Reymond, Le Bernin, Paris o. J.

Nicole Riegel, Die Chigi-Kapelle in Santa Maria del Popolo: eine kritische Revision, in: Marburger Jahrbuch für Kunstwissenschaft, 30. 2003, S. 93-130.

Michael Rohlmann, Michelangelos "Jonas". Zum Programm der Sixtinischen Decke, Weimar 1995.

Erich Schleier, "Lanfrancos Malereien der Sakramentskapelle in S. Paolo fuori le mura in Rom: das wiedergefundene Bild des , Wachtelfalls «, in: Arte Antica e Moderna 29-32, 1965, S. 62-81, 188-201, 343-364.

-, "Lanfrancos ,Elias und der Engel und der Bilderzyklus der Sakramentskapelle von San Paolo fuori le mura in Rom«, in: Bulletin van het Rijksmuseum 18, 1970, S. 3-33, 42 43.

Berthold Schmitt, Giovanni Lorenzo Bernini. Figur und Raum, St. Ingbert 1997.

Sabine Schulze, "Antikes Vorbild in barockem Pathos. Berninis Daniel in der Löwengrube", in: Antikenrezeption im Hochbarock, hg. v. Herbert Beck, Sabine Schulze, Berlin 1989, S. 173-191.

John Shearman, "The Chigi Chapel in S. Maria del Popolo", in: Journal of the Warburg and Courtauld Institutes 24, 1961, S. 129-160.

-, „Pentimenti in the Chigi Chapel «, in: Art the Ape of Nature. Studies in Honor of H. W. Janson, hg. v. Moshe Barasch, Lucy Freeman Sandler, Patricia Egan, New York 1981, S. 219-222.

Wolfgang Stechow, "Heemskerck, the Old Testament, and Goethe«, in: Master Drawings 2, 1964, S. 37-39.

Christina Strunck, "Lorenzo Onofrio Colonna, der römische Sonnenkönig. Neue Dokumentenfunde zu Bernini und seinem Kreis im Archivio Colonna«, in: Zeitschrift für Kunstgeschichte 61, 1998, S. 568-577.

-, "Eine radikale Programmänderung im Palazzo Vecchio. Wie Michelangelos sieger auf Giambologna und Vasari wirkte", in: Michael Rohlmann, Andreas Thielemann (Hg.), Michelangelo. Neue Beiträge, München, Berlin 2000, S. 265-297.

Christina Strunck, "Bellori und Bernini rezipieren Raffael. Unbekannte Dokumente zur Capella Chigi in Santa Maria del Popolo«, in: Marburger Jahrbuch für Kunstwissenschaft 30 (2003), S. 131-182.

Christof Thoenes, "Bernini architetto tra Palladio e Michelangelo", in: Gian Lorenzo Bernini Architetto e l'architettura europea del Sei-Settcento, hg. v. Gianfranco Spagnesi, Marcello Fagiolo, Florenz 1983, Bd. 1, S. 105-134.

Giorgio Vasari, Le vite de' più eccellenti pittori scultori ed architettori scritte da Giorgio Vasari pittore aretino con nuove annotazioni e commenti di Gaetano Milanesi, 8 Bde., hg. v. Gaetano Milanesi, Florenz 1906, Reprint Florenz 1981.

Caterina Volpi, "Salvator Rosa e Carlo De Rossi«, in: Storia dell'arte 93/94, 1998, S. 356373.

Christoph Weber, Genealogien zur Papstgeschichte (Päpste und Papsttum, Bd. 29,1-29,4), Stuttgart 1999-2001.

Kathleen Weil-Garris Brandt, "Cosmological Patterns in Raphael's Chigi Chapel in S. Maria del Popolo", in: Raffaello a Roma. Il convegno del 1983 (Kongreßakten Rom 1983), Rom 1986, S. 127-158. 
Robert Henry Westin, Antonio Raggi: A Documentary and Stylistic Investigation of His Life, Work and Significance in Seventeenth-Century Roman Baroque Sculpture, Diss. Pennsylvania State University 1978.

Rudolf Wittkower, Gian Lorenzo Bernini. The Sculptor of the Roman Baroque, London 1955.

-, Bernini. The Sculptor of the Roman Baroque, 4. Aufl., London 1997.

Marc Worsdale, "Daniel in the Lions' Den«, "Habakkuk and the Angel«, in: Catherine Johnston, Gyde Vanier Shepherd, Marc Worsdale, Vatican Splendour. Masterpieces of Baroque Art (Ausst. Kat. Ottawa 1986), Ottawa 1986, Kat. Nr. 21 u. 22, S. 90-93.

Kaspar Zollikofer, Berninis Grabmal für Alexander VII. Fiktion und Repräsentation, Worms 1994. 